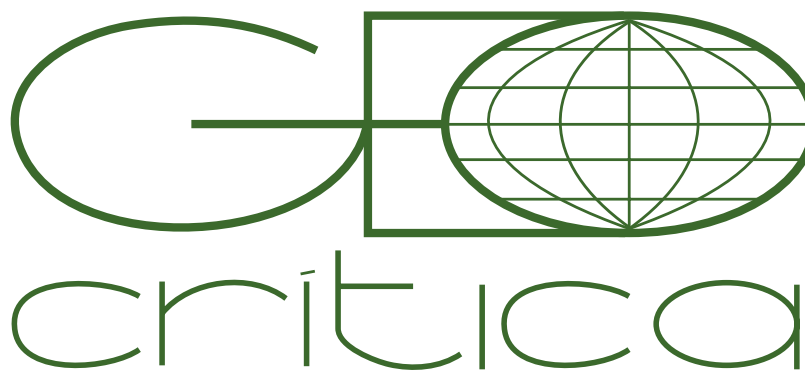

\section{Scripta Nova}

Revista Electrónica de Geografía y Ciencias Sociales Universitat de Barcelona

1 de noviembre de 2018

\title{
DIFERENCIACIÓN SOCIO-ESPACIAL URBANA: MAPA SINTÉTICO DE LA ESTRATIFICACIÓN DE LOS BARRIOS DE BILBAO
}

\author{
Amaia Altuzarra Artola \\ amaia.altuzarra@ehu.eus \\ Irantzu Álvarez González \\ irantzu.alvarez@ehu.eus \\ Elena Martínez Tola \\ elena.martineztola@ehu.eus \\ Arantxa Rodríguez Álvarez
arantxa.rodriguez@ehu.eus
}

Universidad del País Vasco-Euskal Herriko Unibertsitatea (UPV/EHU)

Recibido: 24.1.18; devuelto para correcciones: 7.4.18; aceptado: 24.5.18

\begin{abstract}
Diferenciación socio-espacial urbana: mapa sintético de la estratificación de los barrios de Bilbao (resumen)

Este trabajo presenta una metodología de análisis de la estratificación socio-espacial de barrios que permite discriminar el efecto combinado de distintas dimensiones analíticas de la diversidad de los barrios aplicando técnicas multivariantes: el Análisis Factorial Múltiple (AFM) y el Análisis Clúster. La primera técnica permite fijar las posiciones relativas de los barrios en relación a distintos grupos de variables o dimensiones mientras que la segunda permite obtener agrupaciones de barrios con características homogéneas teniendo en cuenta la influencia de todas las variables de forma conjunta. Utilizando Bilbao como caso de estudio, el trabajo realiza una caracterización de los barrios de la ciudad en función de un conjunto de variables demográficas, socio-económicas, urbanísticas y de cohesión social relativas al año 2011 revelando un mapa sintético de la estratificación socio-espacial de la ciudad.
\end{abstract}

Palabras Clave: Análisis Factorial Múltiple, Análisis Clúster, políticas urbanas, Bilbao.

Urban socio-spatial differentiation: mapping neighborhood stratification in Bilbao (abstract)

This paper proposes an innovative methodology for analysing socio-spatial stratification of neighbourhoods discriminating for the combined effect of various analytical dimensions of neighbourhood differentiation using multivariate statistical techniques: Multiple Factor Analysis (MFA) and Cluster Analysis. The first technique makes it possible to fix the neighbourhoods' relative positions in relation to various groups of variables or dimensions while the second defines groups of neighbourhoods with homogeneous characteristics based on the combined effect of all variables. Using the case of Bilbao, this research presents a characterization of the city's neighbourhoods according to a series of demographic, socioeconomic, cohesion and housing variables projecting a synthetic map of Bilbao's neighbourhood stratification.

Keywords: Multiple Factorial Analysis, Cluster Analysis, urban policy, Bilbao. 
La profundización de los procesos de globalización de las últimas décadas, en combinación con la reciente crisis económica, ha impulsado importantes transformaciones en la estructura, organización y articulación interna de las ciudades de todo el mundo 1 . Junto a estos procesos, la recomposición de los mercados de trabajo y el retroceso brutal de las estructuras de provisión social impuestas por las políticas austeritarias en la última década puntean las principales tendencias de cambio en las áreas urbanas. Todos estos cambios, de manera combinada, han producido nuevas fracturas y desigualdades intra-urbanas a distintas escalas espaciales (ciudades, distritos, barrios) $)^{2}$. En este contexto, se hace necesario formular nuevas aproximaciones al estudio de las realidades urbanas y de los procesos que afectan de manera diferenciada a los barrios de la urbe, procesos que siguen dinámicas diversas y que se muestran no sólo como unidades de intervención sino como agentes sociales que contribuyen de manera decisiva a la transformación de la ciudad 3 .

Por su peso específico para impulsar dinámicas de recomposición socio-espacial, destacan algunos ámbitos en los que los cambios han sido de mayor calado. Así, en el ámbito demográfico, se pueden señalar como fundamentales los cambios en el crecimiento vegetativo de la población, las estructuras familiares y la mayor presencia de población extranjera en las ciudades españolas ${ }^{4}$. En el ámbito urbanístico, la fuerte expansión inmobiliaria de finales de los 90 y principios de los 2000 ha sido uno de los factores claves de la transformación urbana de este periodo. Pero, junto al espectacular aumento del stock inmobiliario y la dimensión física de las ciudades, se han dado también dinámicas significativas de recualificación urbana a diferentes escalas relacionadas con la calidad del parque residencial y el régimen de propiedad de las viviendas que se acompañan también de cambios económico-funcionales cada vez más intensos y veloces. Estos procesos revierten, a su vez, en el funcionamiento de los mercados locales de trabajo con impactos diferenciados sobre su población ${ }^{5}$. Por último, los cambios sociales en curso hacen que las fronteras espaciales entre grupos de población estén relacionadas de forma creciente con las oportunidades de tener un empleo y que este sea estable y de calidad 6 .

En el marco de estos cambios, las desigualdades espaciales dentro de la ciudad han modificado sus características y el modelo tradicional, que divide la ciudad en porciones diferentes según su distancia relativa al centro, ha sido ampliamente superado y sustituido por una estructuración basada en un centro y diferentes tipos de periferias, pudiéndose establecer jerarquías entre las mismas en base a la combinación de diversas características socio-económicas? ${ }^{7}$. Por ello, resulta impor-

1 Caravaca et al., 2017; Guevara, 2015; Méndez et al., 2015.

2 Marcos y Mera, 2011.

3 De las Rivas et al., 2017; Van Kempen, Bolt y Van Ham, 2016.

4 Reques, 2011.

5 Méndez y Prada, 2014; Rubiales, 2016.

6 Pratschke y Morlicchio, 2012; Tezanos, 2001.

7 Leal y Domínguez, 2008. 
tante conocer en detalle la trayectoria de las nuevas dinámicas de diferenciación y, también, hasta qué punto se pueden establecer relaciones entre los distintos procesos en curso, lo que sugiere la necesidad de avanzar en un análisis en profundidad de la información disponible utilizando herramientas estadísticas más sofisticadas y nuevos marcos interpretativos. En definitiva, se trata de abordar el estudio simultáneo de los distintos indicadores que marcan las tendencias latentes, tal y como se viene haciendo en distintos estudios realizados para diferentes ciudades y áreas metropolitanas a nivel europeo y español. Además, el desarrollo económico y social de las ciudades depende en buena medida de las estrategias de planificación y ordenación urbana, para las cuáles los métodos de análisis multivariante y los Sistemas de Información Geográfica (SIG) se han convertido en herramientas fundamentales para la toma de decisiones ${ }^{8}$.

En este marco, el objetivo de este trabajo es es presentar una metodología de análisis de la estratificación socio-espacial de barrios que permite discriminar el efecto combinado de distintas dimensiones analíticas de la diversidad de los barrios. Esta metodología consiste en elaborar un mapa sintético de la estratificación socio-espacial aplicando técnicas multivariantes: el Análisis Factorial Múltiple (AFM) y el Análisis Clúster. La primera técnica permite fijar las posiciones relativas de los barrios en relación a distintos grupos de variables o dimensiones mientras que la segunda permite obtener agrupaciones de barrios con características homogéneas teniendo en cuenta la influencia de todas las variables de forma conjunta. Utilizando Bilbao como caso de estudio, el trabajo realiza una caracterización de los barrios de la ciudad (Figura 1) en función de un conjunto de variables demográficas, socio-económicas, urbanísticas y de cohesión social relativas al año 2011 . El resultado del análisis multivariante es un conjunto de agrupaciones de los barrios de Bilbao que comparten problemáticas como resultado de la combinación ponderada de factores diferenciados, reflejando un mapa sintético de la estratificación socio-espacial de la ciudad que mejora el conocimiento de las estructuras subyacentes en la ciudad y puede contribuir a definir intervenciones ajustadas a las especificidades de cada barrio o grupo de barrios.

En el siguiente apartado de este trabajo, se presenta el marco teórico orientado a la explicación de las principales tendencias en la diferenciación socio-espacial de las ciudades en las últimas décadas así como de los factores determinantes de dichos procesos. En el segundo apartado, se describe pormenorizadamente la metodología empleada y la selección de las variables para el estudio. A continuación, en el tercer epígrafe, se detallan los resultados obtenidos del análisis factorial múltiple. En el cuarto apartado, se recoge el resultado del análisis clúster llevado a cabo a partir de la información basada en los primeros factores extraídos en la aplicación de la técnica multivariante, lo que permite agrupar los barrios en función de características homogéneas. A continuación, en la quinta sección, se proyectan los resultados obtenidos sobre el plano de la ciudad, dando lugar a un mapa de la estratificación

8 Buzai, 2003; Buzai y Baxendale, 2013. 
socio-espacial de los barrios para cada una de las dimensiones estudiadas. Para finalizar, se recogen las conclusiones principales del trabajo, destacándose las oportunidades que brindan las técnicas estadísticas multivariantes para avanzar en el conocimiento de las estructuras urbanas.

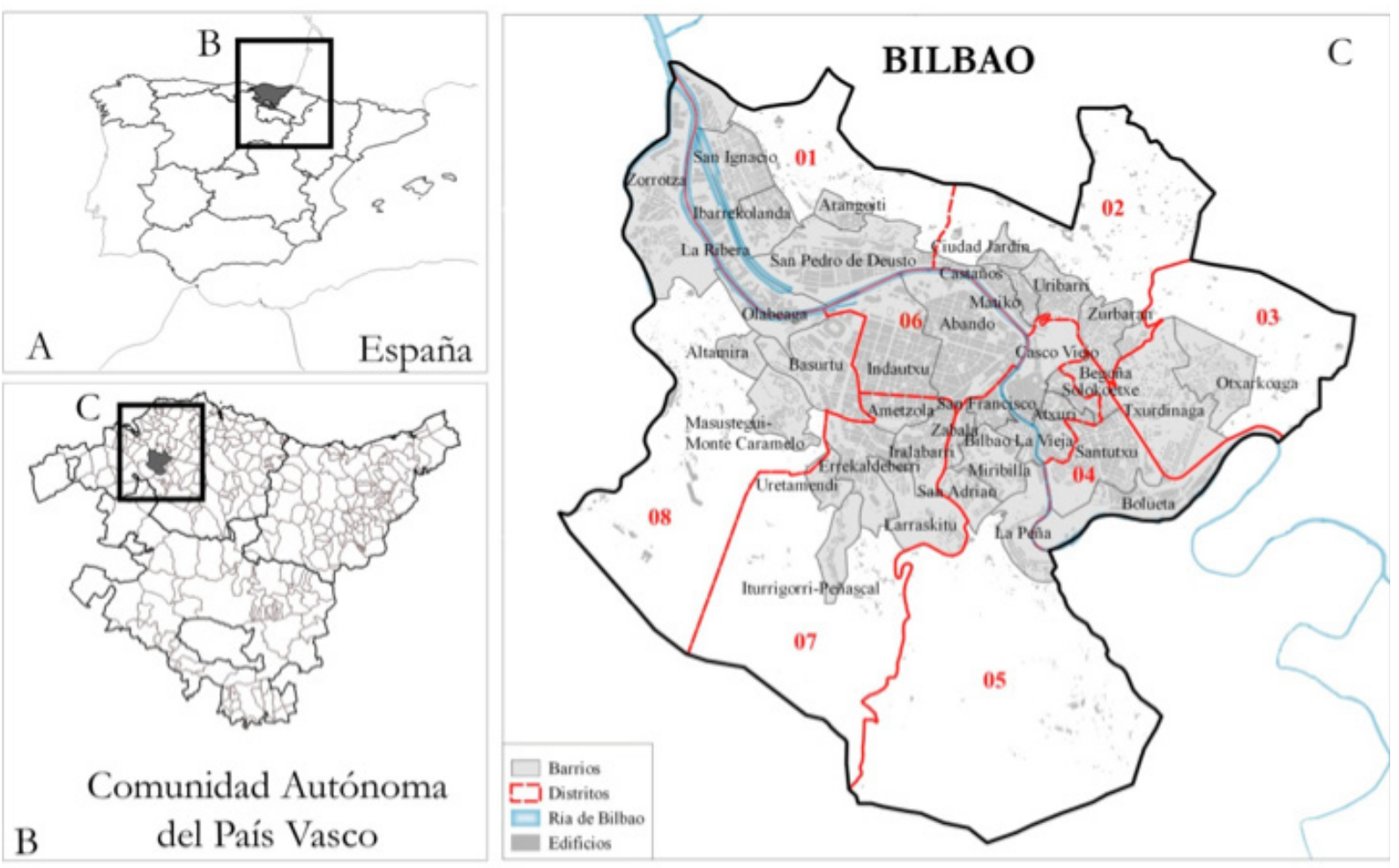

Figura 1: Mapa de situación de Bilbao

Nota: Los distritos administrativos de Bilbao son los siguientes: Distrito 01: Deustu compuesto por los barrios de San Ignacio, Elorrieta, Ibarrekolanda, San Pedro de Deustu, La Ribera y Arangoiti.; Distrito 02: Uribarri compuesto por Castaños, Matiko; Ciudad Jardín, Uribarri, Zurbaran y Arabella; Distrito 03: Otxarkoaga/Txurdinaga integrado por los barrios de Otxarkoaga y Txurdinaga; Distrito 04: Begoña formado por Begoña, Santutxu y Bolueta; Distrito 05: Ibaiondo integrado por Casco Viejo, Iturralde, Solokoetxe, Atxuri, La Peña, Bilbao La Vieja, San Francisco, Zabala, San Adrián y Miribilla; Distrito 06: Abando compuesto por Abando e Indautxu; Distrito 07: Rekalde compuesto por Ametzola, Iralabarri, Errekaldeberri, Larraskitu, Uretamendi eIturrigorri-Peñascal; Distrito 08: Basurtu/Zorrotza formado por los barrios de Basurtu, Olabeaga, Masustegi, Monte Caramelo, Altamira y Zorrotza.

\section{Globalización, políticas urbanas y diferenciación socio-espacial en la ciudad}

A lo largo de las últimas cuatro décadas, los procesos de reestructuración socio-económica que acompañan el fin del Fordismo y la transición hacia un nuevo régimen de acumulación globalizada han tenido en las áreas urbanas un protagonista de excepción. En efecto, la transformación radical de las condiciones de producción y demanda que impulsaron el crecimiento urbano-industrial desde finales de la segunda guerra mundial hasta mediados de los 70, abre paso a una recomposición económica, política y social que altera de forma notable las jerarquías territoriales 
y redefine la forma, función y estructura de los espacios urbanos. Las ciudades, territorios privilegiados de la industrialización Fordista, reemergen, tras la crisis, convertidas en nodos centrales de la nueva economía globalizada, cuya mayor o menor fortuna está fuertemente condicionada por su capacidad para insertarse en las redes de los flujos económicos, tecnológicos y políticos globales ${ }^{9}$.

En el nuevo escenario competitivo global, el declive de los sectores industriales tradicionales y el creciente protagonismo de los servicios, especialmente los servicios avanzados característicos de la denominada economía del conocimiento, apuntalan la recomposición de la base productiva urbana y de las funciones económicas de las ciudades a diferentes escalas. Los cambios en los mercados laborales que acompañan estos procesos de reestructuración económica, en particular la progresiva quiebra de la relación salarial Fordista y la precarización del empleo, han favorecido la aparición de dinámicas de exclusión social y el aumento de la desigualdad que se han convertido en parte integral del contexto urbano ${ }^{10}$. La disminución de los empleos industriales estables y bien remunerados, característicos de la era Fordista, avanza durante este periodo en paralelo a un aumento significativo de la demanda de empleos terciarios de baja cualificación por un lado y de muy alta cualificación por otra, una tendencia que ha sido interpretada en términos de polarización social y que se manifestaría con especial intensidad en las ciudades y regiones metropolitanas más integradas en la economía global ${ }^{11}$.

Paralelamente, algunos autores y/o autoras defienden que la principal tendencia en los procesos de polarización social se asocia a un aumento diferencial de la demanda de las ocupaciones de mayor cualificación respecto a las de baja cualificación, es decir, la profesionalización. A partir de aquí, el debate se centra en las dinámicas que afectan a la evolución de los empleos de menor cualificación en las ciudades a partir de la década de los 80. Estudios realizados con posterioridad plantean que en las ciudades europeas las tendencias del mercado laboral han favorecido la exclusión social más por la profesionalización que por la polarización ${ }^{12}$ debido, entre otras razones, a la menor inmigración y mayor regulación de los mercados laborales que hace que la tesis de la polarización encaje mejor con la realidad urbana norteamericana que con la europea ${ }^{13}$. Con todo, la metamorfosis del empleo aparece como el principal vector de producción de nuevas formas de fragmentación y segregación social y territorial en las áreas urbanas de todo el mundo, dinámica que ha sido capturada por términos como la ciudad "dual"14,

9 Sassen, 1999; Friedman, 1996; Veltz, 1999; Castells, 2010; Marcuse y Van Kempen, 2000.

10 Madanipour et al, 1998; Mingione, 1996; Pacione, 1997; Hamnett, 1994.

11 Sassen, 1991 y 1998; Hamnett, 1994.

12 Van Hamme, 2010.

13 Hamnett, 1994.

14 Mollenkopf y Castells, 1991. 
"polarizada"15, "dividida"16, "cuarteada"17, etc.

A pesar de la enorme variabilidad en cuanto a la intensidad y la periodización del despliegue de los procesos de fragmentación socio-espacial, numerosos estudios reflejan que estas tendencias son comunes a la mayoría de las ciudades europeas ${ }^{18}$. Van Hamme (2010), por ejemplo, muestra que la polarización social ha aumentado en casi todas las ciudades analizadas en las últimas décadas, si bien el nivel de polarización de una ciudad depende, por una parte, del contexto nacional y, por otra, del grado de inserción en la economía global y las nuevas formas de crecimiento económico asociados con la economía basada en el conocimiento. Los principales motores de estos procesos de polarización social en las ciudades europeas están relacionados con la dinámica económica, especialmente los mercados de trabajo en términos de cualificación, la evolución socio-demográfica, la evolución de los mercados inmobiliarios, y las políticas públicas tanto del Estado como de la propia ciudad.

Ahora bien, en las economías industriales avanzadas, las nuevas divisiones socio-espaciales se han visto reforzadas por la reorganización del estado social Keynesiano y el despliegue prolongado de políticas neoliberales que acompaña la transición post-Fordista. En este contexto, las medidas económicas centradas en el control de la inflación y del gasto público se han traducido, a lo largo de las últimas décadas, en la reducción y debilitamiento de las estructuras sociales y de bienestar, provocando la desprotección de sectores de la población cada vez más amplios. Durante este periodo, se acentúa la tendencia hacia una mayor desigualdad en la distribución de la renta que lleva a una concentración creciente de la renta y la riqueza en las capas superiores de la sociedad mientras que la participación de las rentas del trabajo en la renta total disminuye y las capas con menores ingresos de la sociedad ven empeorar su situación relativa ${ }^{19}$. Más recientemente, la tendencia a una mayor desigualdad y exclusión social se ha visto agravada por nuevas dinámicas que se caracterizan por "un fuerte crecimiento del número de personas, empresas y lugares expulsados de los órdenes sociales y económicos centrales de nuestro tiempo"20, que incluyen el empobrecimiento de las clases medias en los países ricos, personas en situaciones de desempleo de larga duración y la expulsión de las y los trabajadores de bajos salarios y desempleados de los programas de salud, bienestar social y/o ayudas por desempleo. Se trata de una situación cualitativamente diferente a las anteriores en la medida en que el sistema ya no tendría interés en incluir a estas personas en su dinámica. El aumento de la exclusión social y la desigualdad se consolida así como un elemento estructural, sistémico, de la nueva realidad socio-económica global, nacional y local.

15 Sassen, 1991.

16 Fainstein, Gordon y Harloe, 1992.

17 Marcuse, 1989.

18 Moulaert et al., 2003; Musterd et al., 2017; Van Hamme, 2010; Tammaru et al., 2015

19 Piketty, 2014.

20 Sassen, 2015:11. 
Por otra parte, en las ciudades y regiones metropolitanas, los cambios en las formas de intervención pública también han contribuido al aumento de la fragmentación y segregación socio-espacial asistidas por el auge de las Nuevas Políticas Urbanas $^{21}$. El desplazamiento de las prioridades de intervención hacia el crecimiento económico y la reestructuración competitiva y el enfoque proactivo y empresarial de las mismas, junto con las nuevas formas de gobernanza urbana, han propiciado el realineamiento productivista de las políticas urbanas que se manifiesta en una agenda política de las ciudades en sintonía con las tendencias apuntadas. Así, en lugar de amortiguar los impactos de las reestructuraciones sociales, políticas y económicas, las Nuevas Políticas Urbanas han sido parte integral de dichos procesos, de modo que las nuevas realidades territoriales son fiel reflejo de las dinámicas de diferenciación, elitización y exclusión socio-espacial. En este sentido, Moulaert et al. (2001) subrayan, en particular, que las tendencias a una mayor fragmentación y segregación socio-espacial en las ciudades europeas se han visto agravados por el despliegue de las Nuevas Políticas Urbanas, específicamente por las estrategias neoliberales de regeneración urbana, que han favorecido dinámicas de revitalización a dos velocidades en diversas ciudades europeas.

En el caso español también es posible identificar estas tendencias en diferentes estudios realizados en la última década. Así, Leal y Domínguez (2008) destacan, en el caso de Madrid, la existencia de un mayor distanciamiento social y económico, incluso antes de la crisis, en un contexto marcado por la bonanza económica, lo que se explica principalmente por una mayor segregación de las clases mejor situadas tanto en las periferias como en el propio centro de la ciudad. A partir del año 2007, se observa también una importante fragmentación entre distintos sectores de la ciudad que se traducen, entre otros, en grandes diferencias en términos de desempleo y una mayor segregación socio-espacial, explicada en parte por el aumento de los privilegios de las clases superiores junto con el mayor impacto de las políticas de austeridad en la población con peores condiciones socio-económicas ${ }^{22}$. Igualmente, se identifica una tendencia en la que el funcionamiento del mercado inmobiliario, por una parte, y el apoyo presentado por el planeamiento público, por otra, ha favorecido la atracción de los grupos sociales mejor situados hacia determinadas áreas (periféricas y centrales), ahondándose de esta manera en la citada fragmentación ${ }^{23}$.

En Barcelona, también se produce un incremento de la segregación urbana y un aumento de la polarización en la primera década de los 2000. Este proceso pone de manifiesto que son los grupos mejor situados los que muestran una mayor tendencia a situarse en barrios segregados ${ }^{24}$. Asimismo, se distingue un proceso de cambio en la población de la ciudad de forma que el centro va siendo progresivamente ocupado por los grupos mejor situados, lo que unido al hecho que se ve afectado por la

21 Moulaert et al., 2003: Rodríguez et al., 2001.

22 Leal y Sorando, 2016.

23 Méndez y Prada, 2014.

24 Donat et al., 2014; Rubiales et al., 2012. 
crisis en menor medida, puede contribuir a que estas áreas presenten un comportamiento relativamente mejor que el resto de la ciudad ${ }^{25}$.

En el caso del área metropolitana de Valencia, las condiciones socio-económicas de la población constituyen el principal factor explicativo de la estructura territorial en la primera década de los 2000, ganando en relevancia tras la crisis de 2007. También aumentan los contrastes territoriales, siendo la inmigración el factor explicativo principal de algunas de las tendencias asociadas más relevantes ${ }^{26}$. Cabe resaltar que son numerosos los análisis urbanos que se centran en la ubicación y segregación socio-espacial de la población inmigrante; no obstante, en el caso de algunas ciudades estatales, la población extranjera no ha sido especialmente elevada, de manera que es muy difícil establecer relaciones claras, aunque sí puede identificarse los lugares de mayor concentración ${ }^{27}$.

Los estudios realizados en ciudades andaluzas constatan también la importante relación que se establece entre la estructura social y la segregación en el espacio urbano. En el caso de Málaga, se identifica para el año 1991 una tendencia que posteriormente se confirma en diversos estudios: "son los de mayor condición quienes más se segregan de los que tienen menos, y no al revés, como a veces parece" 28 . En Sevilla, por su parte, en la década de los 80 y 90, se constata un incremento de la polarización socio-espacial en la medida en la que existe una trayectoria que hace que las diferentes áreas urbanas se alejen de la media de la ciudad en lo que a los indicadores de estatus social se refiere, al tiempo que se agrupan en torno a zonas que comparten valores similares ${ }^{29}$.

En el caso de Bilbao, las últimas décadas han sido un periodo de cambios profundos económicos, sociales y urbanísticos que la han convertido en un referente de éxito y buenas prácticas urbanísticas. Sin embargo, diversos trabajos previos realizados en la ciudad de Bilbao y su área metropolitana ${ }^{30}$ resaltan el carácter eminentemente físico y localizado de la política de regeneración urbana implementada durante este periodo. De hecho, en la década de los noventa, la ciudad muestra un aumento de las diferencias en términos de segregación residencial y socio-espacial, en lo que parece una tendencia que ahonda en el viejo esquema centro-periferia ${ }^{31}$. Pero, al margen de valoraciones más o menos ponderadas, la crisis financiera y las políticas austeritarias de la última década también han tenido, al igual que en otras ciudades de su entorno, importantes consecuencias, especialmente sobre los secto-

25 Rubiales, 2016.

26 Salom y Fajardo, 2017.

27 Este sería el caso de Bilbao, donde el estudio de la segregación residencial de la población inmigrante realizado por Lavín (2009) evidencia su escasa presencia relativa en la ciudad y la mayor concentración de esta población en los barrios de Bilbao La Vieja y San Francisco.

28 Del Pino, 2001: 139.

29 Díaz, 2010.

30 Esteban, 2000; Rodríguez y Martínez, 2001; Gómez, 1998; Plaza, 2000; Vicario y Martínez, 2003; Rodríguez, Martínez y Guenaga, 2001; y Antolín, Fernández y Lorente, 2010, entre otros. 31 Rodríguez et al., 2001; Antolín et al., 2010. 
res más vulnerables, con una disminución de las rentas personales, precarización del empleo, aumento de la población en situaciones de riesgo o mayores dificultades en el acceso a la vivienda y a los servicios colectivos. Estos cambios se superponen sobre las estructuras de segregación socio-espacial preexistentes de modo que su impacto no se da con igual intensidad y forma en toda la ciudad sino que, por el contrario, varía significativamente de un barrio a otro, dependiendo de sus características y mayor o menor grado de vulnerabilidad ${ }^{32}$. Así pues, las características de la población (envejecimiento, nivel formativo, presencia grupos sociales de riesgo, etc.), la estructura y dinámica del sector inmobiliario, que condiciona el acceso a la vivienda y la calidad de ésta, el nivel de renta, o la ocupación de la población, son variables determinantes a la hora de entender la mayor o menor capacidad de resistencia de los barrios ante la crisis económica ${ }^{33}$. Por tanto, identificar la influencia relativa que los diferentes elementos han tenido sobre los procesos de segregación y estratificación socio-espacial en los barrios de la ciudad no es una tarea simple, por lo que resulta relevante el estudio de los análisis realizados en otras áreas urbanas españolas para tratar de identificar algunos patrones comunes.

En resumen, la segregación socio-espacial en las ciudades estatales se consolida como dinámica estructural a lo largo del tiempo, independientemente de la evolución económica general y apoyada por las intervenciones urbanísticas de las últimas décadas. Además, debido a la profesionalización del empleo, así como a los desiguales efectos de la crisis sobre los diferentes grupos sociales, se identifica con nitidez una tendencia a la polarización socio-espacial basada en gran medida en la tendencia de los grupos mejor situados a diferenciarse espacialmente del resto, bien en base a la ocupación de nuevas promociones como a la gentrificación de barrios en el centro de las ciudades. En el caso de la población inmigrante, se tiene en cuenta de manera creciente en el análisis de la segregación socio-espacial de las ciudades, aunque en cada vez más casos se considera como una categoría independiente de análisis con incidencia particular en la articulación del espacio urbano ${ }^{34}$.

Tal y como se ha presentado, son numerosos los factores que interactúan a la hora de definir los procesos de segregación sociespacial identificables a escala urbana y, por ello, resulta importante avanzar en el establecimiento de mecanismos que hagan posible identificar los elementos que caracterizan la situación relativa de unos lugares de la ciudad respecto a otros, no solo a nivel agregado, sino también para cada una de sus dimensiones. Este conocimiento resulta especialmente relevante para la puesta en marcha de iniciativas que busquen resolver la problemática urbana en sus diferentes ámbitos mediante mecanismos participativos en los que las personas y lugares más afectados tengan capacidad de asociación, interlocución y participación en las políticas correspondientes. Es en este sentido en el que se plantea el análisis siguiente en el que no sólo se identifican las diferencias agregadas

32 Méndez et al., 2015, Alguacil et al., 2014.

33 Arias, 2000; Alguacil et al., 2014.

34 Salom y Fajardo, 2017. 
de los espacios, sino que también se profundiza en aquellos ámbitos que les son más o menos semejantes en función de unas dimensiones previamente seleccionadas.

\section{Mapa sintético de la estratificación socio-espacial de los barrios de Bilbao. Metodología estadística y selección de variables}

El objetivo propuesto consiste en elaborar un mapa sintético de la estratificación socio-espacial de los barrios de Bilbao aplicando técnicas multivariantes (Análisis Factorial Múltiple-AFM) y un análisis clúster. De esta manera, el AFM permiten fijar las posiciones relativas de los barrios en relación desde distintas perspectivas, mientras que el análisis clúster complementa el anterior y permite obtener obtienen grupos de individuos (barrios, en este caso) con características homogéneas teniendo en cuenta la influencia de todas las variables de forma conjunta.

\section{Metodología}

El AFM es un método factorial adaptado al tratamiento de datos en las que un mismo conjunto de individuos se representa a través de varios grupos de variables ${ }^{35}$. El objetivo del AFM es definir la estructura subyacente de un conjunto de datos analizando la estructura de correlaciones entre las variables mediante la definición de una serie de dimensiones, denominadas factores. Un factor se define como cualquier combinación lineal de variables en la matriz de datos.

El AFM se basa en la metodología del Análisis de Componentes Principales (ACP) y se compone de dos etapas. En la primera (análisis parcial) se efectúa un ACP normado de cada tabla de datos y se retiene el primer valor propio de cada una de ellas. En la segunda etapa (análisis global) se realiza un ACP de la tabla global que resulta de yuxtaponer todas las tablas, ponderadas por el inverso del primer valor propio obtenido en la etapa anterior. Esta ponderación mantiene la estructura de cada tabla, ya que todas las variables han recibido la misma ponderación, pero consigue equilibrar la influencia de los grupos, ya que la inercia máxima de cada una de las nubes de individuos, definida por los diferentes grupos, vale 1 en cualquier dirección.

El objetivo del AFM es analizar la estructura común de las diferentes tablas de datos, poniendo de relieve cuales son los elementos heterogéneos ${ }^{36}$. El AFM proporciona, además de los resultados clásicos del ACP, medidas globales de relación entre los grupos de variables a través de las matrices de coeficientes RV y Lg, que permiten cuantificar la semejanza global entre los grupos de variables ${ }^{37}$. El AFM es un potente instrumento para generar representaciones gráficas que ayudan a la interpretación de los resultados. Por un lado, el AFM genera la representación gráfica de las variables que permite observar las proximidades entre los puntos-variables

35 Escofier y Pagès, 1990, 1992 y 1994.

36 Ver Escofier y Pagès (1990) y Lebart, Morineau y Piron (1995) para una explicación más detallada de este método estadístico.

37 García y Abascal, 2003. 
de las diferentes tablas. Por otro lado, el AFM obtiene representaciones gráficas de la nube de individuos caracterizados por las variables de cada grupo (individuos parciales) y por el conjunto de variables (individuo medio) sobre el mismo plano factorial. Esta proyección permite identificar la proximidad entre los puntos-individuo de las diferentes tablas y detectar las diferencias. Así, el AFM presenta ventajas analíticas respecto al ACP para el objetivo del trabajo ya que permite aprehender más matices de información disponible. La razón principal es que a menudo las observaciones de un conjunto de variables sobre un grupo de individuos pueden presentar estructuras de comportamiento diferentes debido a que hay que variables que caracterizan distintas dimensiones del conjunto de individuos. Esas diferentes estructuras pueden quedar ocultas en análisis en los que las variables se estudian conjuntamente (ACP). El AFM, por tanto, permite identificar los elementos que caracterizan la situación relativa de los barrios de la ciudad a nivel agregado y para cada una de sus dimensiones.

El AFM se complementa con un Análisis Clúster ${ }^{38}$, que permite agrupar a los individuos en grupos o clases. Los individuos que pertenecen a una misma clase comparten características similares entre ellos y diferentes a las características que definen a los individuos de otro grupo.

Finalmente, todos los resultados obtenidos se han volcado en un Sistema de Información Geográfica para poder analizar los resultados de forma geográfica y explicar en más detalle las dinámicas que se han obtenido del análisis estadístico.

\section{Selección de variables}

Teniendo presente el objetivo de este trabajo, se han seleccionado un conjunto de variables agrupadas en 4 grupos (Cuadro 1), siguiendo las pautas de los estudios realizados al respecto ${ }^{39}$ y de la información disponible. Cada uno de estos grupos caracteriza distintas dimensiones de la diversidad de los barrios de Bilbao, así como áreas potenciales de actuación de las políticas públicas: dimensión demográfica, condición socio-económica, características de la vivienda y cohesión social. Todas las variables incluidas son de naturaleza continua y los individuos estudiados son los cuarenta barrios que conforman el municipio de Bilbao ${ }^{40}$. Los datos sobre población provienen del Padrón Municipal ${ }^{41}$ y el resto de variables se han obtenido a partir del Censo de Población y Viviendas, disponible en Eustat ${ }^{42}$, referidos al año 201 1, con la

38 El software utilizado es el SPAD 7.0.

39 Antolín et al., 2010; Del Pino, 2001; Salom y Fajardo, 2017, entre otros.

40 No se han incluido en el análisis los denominados "barrios diseminados" de Bilbao debido a las deficiencias en la información disponible. Los barrios diseminados son los barrios que integran las afueras del municipio de Bilbao, principalmente zonas muy poco urbanizadas (zonas sin color del mapa de la Figura 5).

41 Datos obtenidos en la página web de Lan Ekintza, entidad perteneciente al Ayuntamiento de Bilbao: http://www.bilbao.eus/cs/Satellite?c=Page\&cid=1272963760362\&locale=3000001694\&pagename=LanEkintza\%2FPage\%2FLKZ_PTPaginaGenerica [17 de Enero de 2018].

42 Eustat - Instituto Vasco de Estadística (www.eustat.eus). Para sintetizar la información referida 
excepción de los datos correspondientes a la renta media personal, provenientes de la Estadística de Renta Personal y Familiar, elaborada por EUSTAT.

El grupo de variables que forman la "dimensión demográfica" está compuesto por 4 variables: población total, población extranjera, densidad de la población total y densidad de la población extranjera. Este grupo de variables pretende capturar las características demográficas de la población de cada barrio y además recoge información sobre el volumen de población que tiene el barrio dentro de la ciudad ${ }^{43}$. El grupo denominado "condición socio-económica" está formado por 3 indicadores: tasa de ocupación, porcentaje de población ocupada en profesiones superiores e intermedias y renta personal media disponible. A través de este grupo de variables se pueden identificar las diferencias inter-barrios asociadas a las características económicas, laborales y profesionales de los habitantes.

Las variables que componen el grupo "características de la vivienda" son: índice de confort de la vivienda, superficie media de la vivienda por individuo y porcentaje de viviendas en régimen de propiedad (pagada o hipotecada). Este grupo de variables recoge información sobre la titularidad y las características físicas de las viviendas.

Finalmente, se ha incluido un grupo denominado "cohesión social" formado por las siguientes variables: porcentaje de personas en ocupaciones no cualificadas, tasa de paro y porcentaje de viviendas en régimen de alquiler. Este grupo de variables permite reflejar una evaluación alternativa de bienestar y se centra principalmente en las posibilidades de la población para tener una mayor movilidad social, inclusión y participación ciudadana ${ }^{44}$. En línea con la OECD (2011), resulta de especial interés identificar los elementos diferenciales relacionados no sólo con los mecanismos de exclusión social sino también con aquéllos relacionados con dinámicas de expulsión que no estarían relacionados con las tradicionales características socio-económicas ${ }^{45}$, de manera que consideramos que la inclusión de las dimensiones socio-económica y de cohesión social de forma separada permite aprehender estas diferencias y recoge matices relacionados con los procesos de exclusión social que no necesariamente están recogidas en la dimensión socio-económica.

\footnotetext{
a las características principales, las instalaciones y los servicios de las viviendas Eustat elabora un índice (Índice de confort, incorporado al estudio), cuyo valor señala aproximadamente el grado de comodidad o bienestar que puede tener una vivienda (para mas detalle ver http://www.eustat. eus/estadisticas/anno_2011/metadata.html).

43 La población total y la población extranjera tienen una correlación alta (superior al 0.7) con el número de establecimientos, así estas variables aportan información sobre el peso relativo del barrio en términos poblacionales y, también, una aproximación a su dimensión productiva. Se ha optado por mantener en el modelo estas variables poblacionales con el objetivo de mantener un equilibro en el número de variables que componen cada grupo y un número de grupos de variables razonable.

44 OECD, 2011; y Van Hamme, 2010.

45 Sassen, 2015.
} 
Cuadro 1: Variables seleccionadas

\begin{tabular}{|c|c|c|c|c|c|}
\hline Grupo & Variables & Media & $\begin{array}{l}\text { Desviación } \\
\text { Estándar }\end{array}$ & Min & Máx \\
\hline \multirow{4}{*}{$\begin{array}{l}\text { Dimensión } \\
\text { demográfica }\end{array}$} & Población total & 8800,0 & 7412,8 & 423 & 32968 \\
\hline & Población extranjera & 742,3 & 604,6 & 25 & 2465 \\
\hline & Densidad población total (hab/km²) & 23556 & 13922 & 592 & 50326 \\
\hline & Densidad población extranjera $\left(\mathrm{hab} / \mathrm{km}^{2}\right)$ & 2437,1 & 2661 & 35 & 14921 \\
\hline \multirow{3}{*}{$\begin{array}{c}\text { Condición } \\
\text { socio-económica }\end{array}$} & Profesiones superiores e intermedias (\%) & 46,2 & 13,9 & 16,6 & 72,8 \\
\hline & Renta personal media & 17670 & 5224 & 9794 & 32572 \\
\hline & Tasa de ocupación & 59,3 & 5,5 & 46,6 & 75,8 \\
\hline \multirow{3}{*}{$\begin{array}{c}\text { Características de las } \\
\text { viviendas }\end{array}$} & Índice de confort & 68,6 & 7,3 & 49,8 & 91,4 \\
\hline & \% Viviendas en propiedad & 84,2 & 2,6 & 76,4 & 87,9 \\
\hline & Superficie de la vivienda por individuo & 31,5 & 4,8 & 22,3 & 44,2 \\
\hline \multirow{3}{*}{ Cohesión social } & \% Trabajadores No-cualificados & 5,4 & 1,7 & 2,2 & 8,7 \\
\hline & Tasa de paro & 18,7 & 4,6 & 10,5 & 31,7 \\
\hline & \% Viviendas en alquiler & 9,8 & 2,6 & 4,9 & 16,6 \\
\hline
\end{tabular}

Fuente: Elaboración propia

De esta manera se ha formado una matriz de 40 individuos (barrios) y 12 variables agrupadas en 4 grupos. La Cuadro 1 presenta las variables incluidas en cada grupo, y sus medidas descriptivas básicas (media, desviación estándar y los valores máximos y mínimos).

\section{Resultados del AFM}

El primer paso en el AFM consiste en estudiar cuáles son las variables más relevantes a la hora de explicar la variabilidad de los grupos. Para ello se ha realizado un análisis global de los datos del que se desprende que existen tres factores principales que explican el $80,5 \%$ de la variabilidad de los datos (Cuadro 2). Este primer análisis pone de manifiesto la existencia de un primer eje o factor global predominante en el sentido de que recoge un importante porcentaje de inercia, un $52,72 \%$ frente al $19,77 \%$ de variabilidad que se proyecta en el segundo factor global. Aunque la tercera componente también recoge una inercia importante, un $7,95 \%$, nuestra atención se va a centrar principalmente en el primer plano factorial generado por las dos primeras componentes, ya que en él se observan las principales características que explican las diferencias entre los barrios $(72,53 \%$ de la varianza). Por otro lado, la relativa proximidad del primer valor propio $(2,9)$ al número de grupos de variables considerados (4 grupos), y el alto porcentaje de la variabilidad total que explica el factor asociado al mismo $(52,76 \%)$, sugieren que este factor es común a los dos grupos.

Cuadro 2: Primeros factores. Análisis global

\begin{tabular}{|c|c|c|c|}
\hline Número & Valor propio & Porcentaje & Porcentaje acumulado \\
\hline 1 & 2,9566 & 52,7618 & 52,7618 \\
\hline 2 & 1,1081 & 19,7738 & 72,5356 \\
\hline 3 & 0,4457 & 7,9542 & 80,4898 \\
\hline
\end{tabular}


La proyección de las variables sobre los planos factoriales permite visualizar la contribución de cada una de las variables a la formación de los factores y el signo de las mismas. En la Figura 2 se proyectan las variables sobre el primer plano factorial y permite observar la correlación entre las variables y los factores 1 y 2 (Figura 2$)^{46}$.

Las variables que más contribuyen a la formación de los ejes son las relacionadas con la condición socio-económica, la cohesión social y las características de la vivienda. Las variables relativas a la condición socio-económica y a las características de la vivienda tienen coordenadas de igual signo positivo (semiplano de la izquierda), lo que se traduce en un efecto talla, muy habitual en el AFM. Puede decirse, por tanto, que este primer eje pone de relieve, de izquierda a derecha, una clasificación general de los barrios de mejor a peor situación socio-económica y de vivienda. La misma lectura puede hacerse de las variables del grupo de cohesión social que aparecen situadas, en este caso, en el semiplano de la derecha.

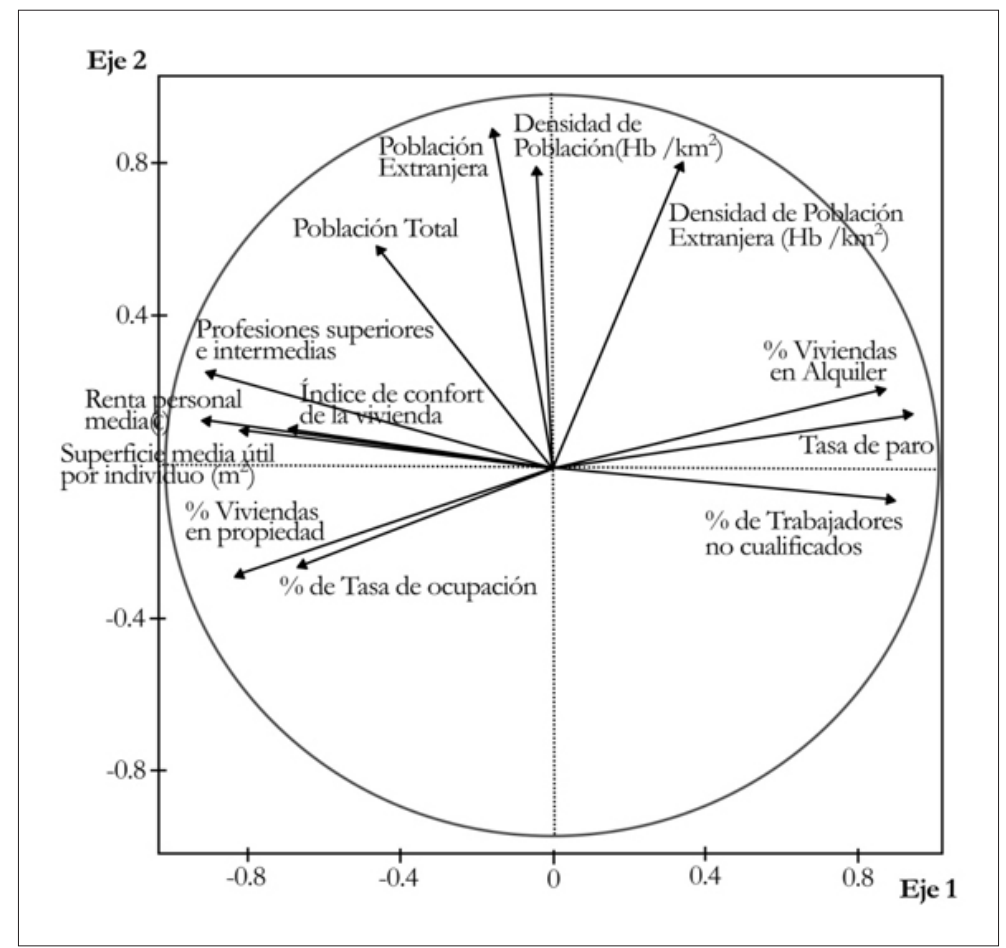

Figura 2: Proyección de las variables activas en el primer plano factorial (1-2)

El segundo factor (Eje 2) pone de manifiesto una segunda caracterización de los barrios en función de la dimensión demográfica. Se observa que todas las variables de este grupo tienen el mismo signo positivo (semiplano superior) permitiendo una clasificación de los barrios de mayor a menor (de arriba a abajo) diversidad cultural y demográfica.

46 La validación de los resultados del AFM se ha realizado mediante el análisis de la intra-estructura e inter-estructura de los grupos de variables. Los resultados de estos análisis están disponibles bajo petición. 
Con base en esta disposición de las variables, en el primer plano factorial (Figura 3) se han proyectado los individuos medios, esto es, la posición media relativa que ocupan los barrios, teniendo en cuenta todos los puntos de vista o grupos de variables considerados.

De acuerdo con la interpretación que hemos realizado de la proyección de las variables, el primer factor refleja, con algunas excepciones que comentaremos posteriormente, el ranking general de barrios en cuanto a la situación socio-económica, la cohesión social y las características de las viviendas. Los barrios con un mayor porcentaje de población que disfrutan de una situación socio-económica favorable y disponen de viviendas en condiciones más favorables son en orden decreciente: Indautxu, Abando, Castaños, Miribilla, Begoña, San Pedro de Deustu, Basurtu e Ibarrekolanda. Los barrios que ocupan los últimos lugares son: Zabala, Bilbao la Vieja, Uretamendi, Otxarkoaga, San Francisco e Iturrigorri-Peñascal.

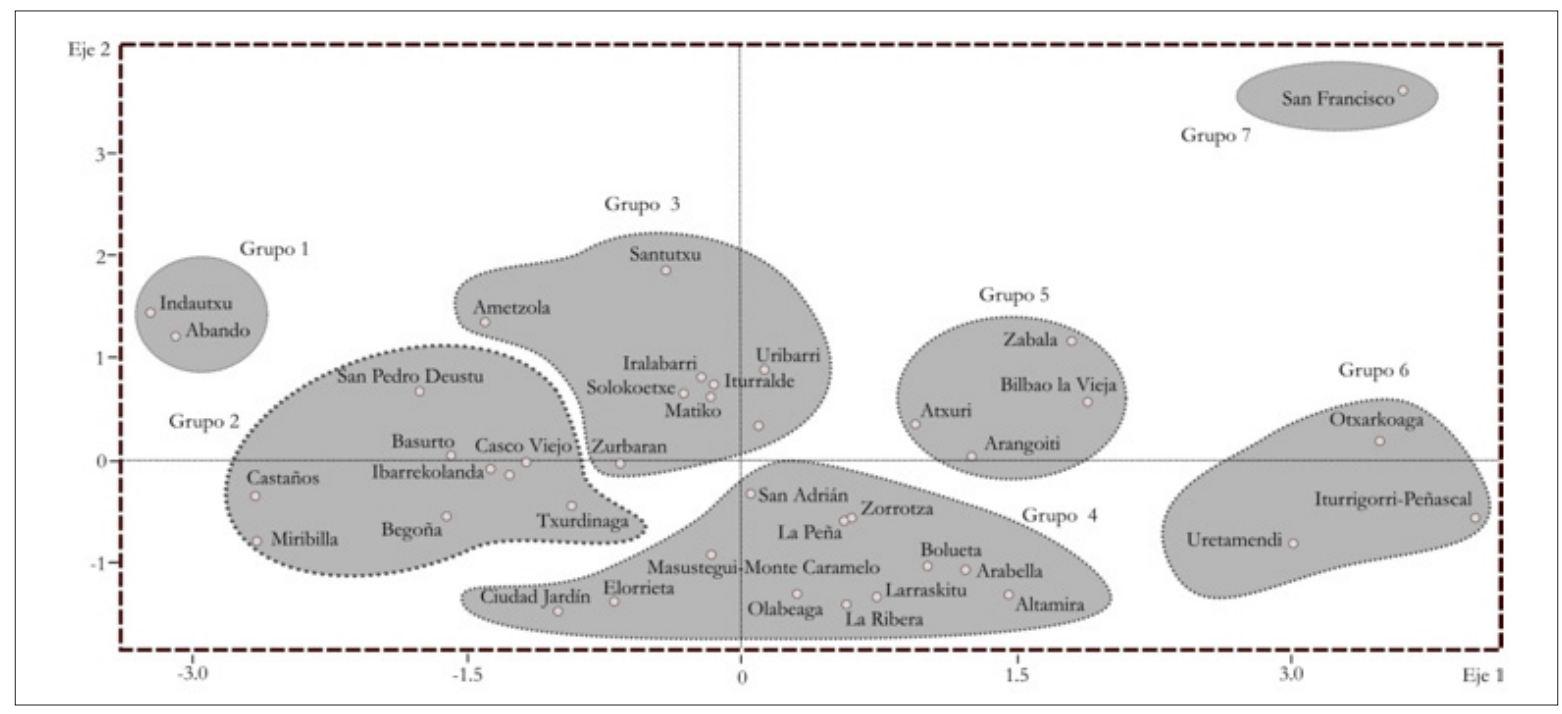

Figura 3: Proyección de los individuos medios en el primer plano factorial (1-2). Clústeres

El segundo eje proporciona información relativa a los rasgos demográficos de los barrios, pudiendo ordenar los barrios de mayor a menor población y densidad de población (total y extranjera). El barrio que concentra mayor porcentaje de población extranjera y mayor densidad de población extranjera es San Francisco ${ }^{47}$.

\section{Análisis Clúster}

El análisis clúster se ha realizado a partir de la información obtenida en el AFM anterior en los tres primeros factores extraídos. La aplicación del análisis clúster permite agrupar a los barrios en siete grupos diferentes. Los barrios que pertenecen a una misma clase tienen características homogéneas en cuanto a sus rasgos

47 San Francisco tiene un comportamiento outlier. Sus características específicas no quedan suficientemente definidas en el plano. 
demográficos, de vivienda y socio-económicos. Los barrios que pertenecen a clases diferentes presentan rasgos distintos. Es preciso señalar que los barrios en el seno de un mismo grupo no comparten necesariamente todas las variables que caracterizan a la clase, aunque sí gran parte de ellas.

En la Figura 4 se presenta el dendograma que resulta de aplicar el método jerárquico-aglomerativo de Ward. Se puede observar cómo partiendo del conjunto de barrios se pueden obtener dos grandes grupos de barrios los cuales a su vez se pueden ir dividiendo en subgrupos. Hemos optado por siete grupos por ser la opción que permitía obtener matices relevantes desde la perspectiva de la ordenación urbana. En la Figura 3, presentada anteriormente, se han agrupado sobre el primer plano factorial los barrios en las 7 clases que se han obtenido del Análisis Clúster y se puede observar que esa agrupación recoge barrios con una posición y, por lo tanto, unas características similares en relación a las variables seleccionadas, lo que consolida la elección de 7 grupos de barrios que expliquen la estratificación socio-espacial de los barrios.

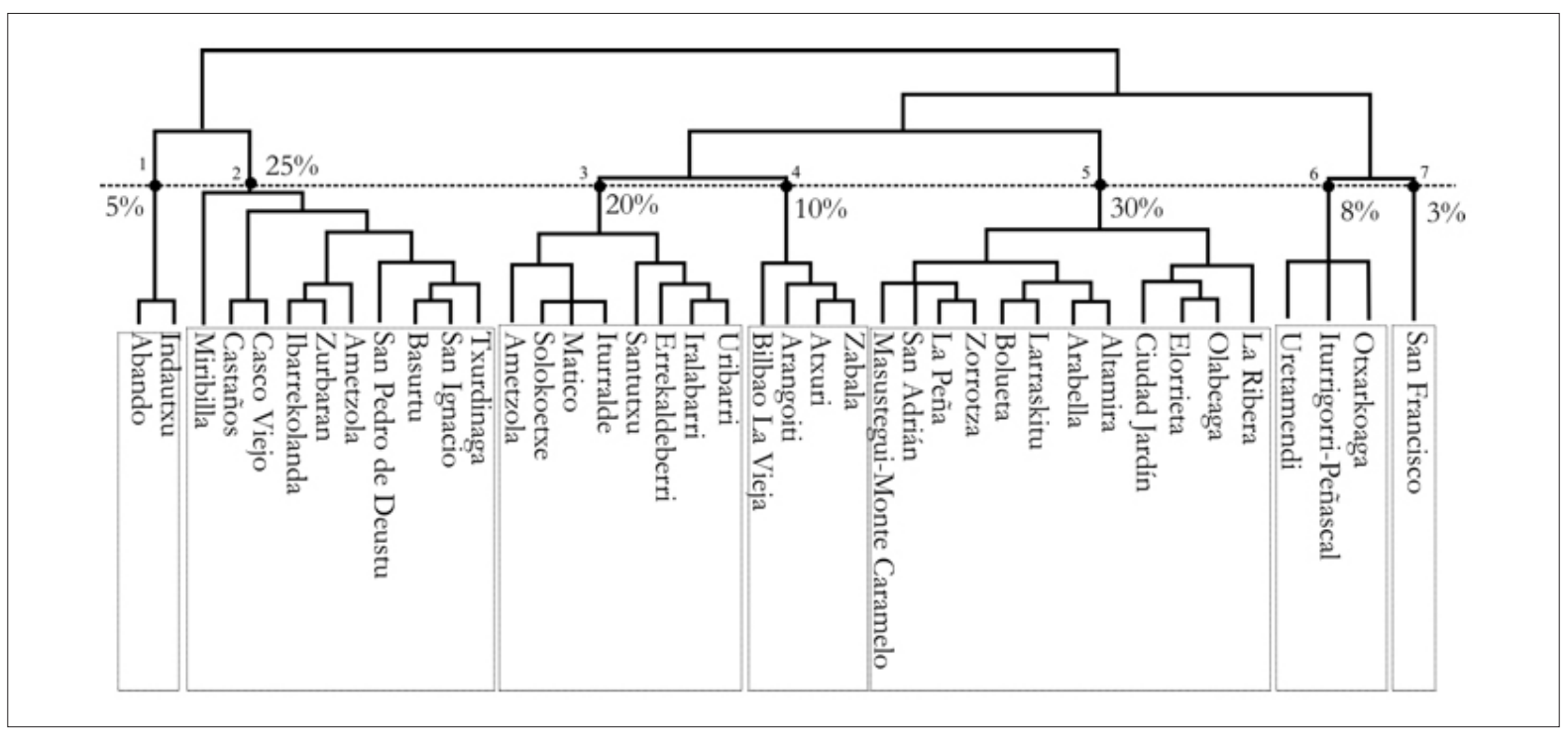

Figura 4: Dendograma

El grupo 1 está formado por dos barrios: Indautxu y Abando. Este grupo, núcleo económico, comercial y turístico de la ciudad, se caracterizan por tener una renta personal media elevada. La renta media en este grupo es de 32.516 euros, casi el doble que la media para el conjunto de los barrios estudiados que es de 17.670 euros. El $72,7 \%$ de la población se dedica a profesiones de rango intermedio o superior (frente a una media del $46,2 \%$ para el conjunto de barrios). Los dos barrios de este grupo concentran una parte importante de la población (residen un total de 52.000 habitantes. Además, son barrios más extensos que la media para Bilbao $\left(1,070 \mathrm{~km}^{2}\right.$ frente a $\left.0,435 \mathrm{~km}^{2}\right)$. La superficie media útil de la vivienda por persona también es significativamente mayor en los barrios de este grupo $\left(42,9 \mathrm{~m}^{2}\right)$ respecto a la media de Bilbao, que es $31,5 \mathrm{~m}^{2}$. Se trata, por tanto, de barrios que destacan por una alta capacidad económica, elevada población y mayor tamaño de sus viviendas. 
El grupo 2 reúne a nueve barrios: Castaños, Miribilla, Begoña, Ibarrekolanda, San Pedro de Deustu, Basurtu, San Ignacio, Txurdinaga y Casco Viejo. Estos barrios comparten algunas características socio-económicas con el grupo anterior. Para este grupo de barrios la renta personal media (21.927€), aunque mayor que la media del universo estudiado, es algo más bajo que para los barrios del grupo 1. Lo mismo sucede con el porcentaje de personas que se dedican a profesiones de nivel intermedio o superior $(59,6 \%)$. El índice de confort de las viviendas de este grupo es superior a la media (75,6 frente al 68,6 para el conjunto de Bilbao). Destaca el elevado porcentaje de viviendas en propiedad: el 86,3\% (frente al 84,3\% que caracteriza a la media de Bilbao) de los hogares que residen en los barrios de este grupo tienen su vivienda en este régimen. La tasa de ocupación es elevada $(62,7 \%$ frente al 59,3\% para el conjunto de los barrios bilbaínos). Son barrios, por tanto, con capacidad socio-económica alta-moderada y con un stock residencial menos envejecido que el grupo 1 .

El grupo 3 aglutina a nueve barrios: Ametzola, Santutxu, Solokoetxe, Zurbaran, Iturralde, Matiko, Iralabarri, Uribarri y Errekaldeberri. La característica más destacada de estos barrios es la relativa alta densidad de población, 40.795 habitantes por km² de media frente a 23.556 habitantes por $\mathrm{km}^{2}$ en el conjunto de Bilbao. En términos absolutos, además, concentran un volumen significativo de población extranjera con una media de 1.098 personas extranjeras frente a las 742 de media en el conjunto de Bilbao. Se trata, por tanto, de un grupo de barrios con elevada densidad demográfica y diversidad cultural y una renta media equivalente a la media de la ciudad.

El grupo 4 agrupa a doce barrios: Ciudad Jardín, La Ribera, Elorrieta, San Adrián, Masustegui-Monte Caramelo, Olabeaga, La Peña, Larraskitu, Bolueta, Arabella, Altamira y Zorrotza. Estos barrios se caracterizan por una menor densidad de población (9.500 habitantes por km2 frente a 23.556 habitantes por $\mathrm{km}^{2}$ en el conjunto del municipio). El porcentaje de población ocupada en profesiones superiores y/o intermedias es inferior a la media $(39,4 \%$ frente a $46,2 \%$ en el conjunto de Bilbao). En este grupo de barrios se concentra un volumen medio de población extranjera menor que en el conjunto de Bilbao. En resumen, son barrios de baja densidad demográfica y con ocupaciones poco cualificadas.

El grupo 5 incluye cuatro barrios: Arangoiti, Atxuri, Zabala y Bilbao La Vieja. Este grupo de barrios se caracteriza por tener una mayor ratio de población extranjera respecto de la población local: 18 residentes extranjeros por cada 100 residentes locales frente a 10 residentes extranjeros por cada 100 residentes locales en el conjunto de Bilbao. La densidad de población extranjera es de 5.109 por km2, mientras que en el conjunto de Bilbao la densidad es de 2.437 habitantes extranjeros por $\mathrm{km} 2$. Por otra parte, destaca el relativamente alto porcentaje de viviendas en alquiler, $12,4 \%$ de las viviendas, comparado con la media del conjunto de la ciudad, $9,9 \%$. Son barrios, en consecuencia, con una relativa alta diversidad demográfica y presencia del régimen de alquiler de vivienda. En el Apéndice (el cuadro 1.A recoge la información detallada sobre cada clase). 
Finalmente, el grupo 6 está formado por tres barrios: Uretamendi, Iturrigorri-Peñascal y Otxarkoaga. Con una tasa de ocupación de la población residente entre 16 y 64 años inferior a la media ( $49,3 \%$ frente al 59,3\%), una tasa de paro superior a la media de la ciudad (28\% frente al $18,6 \%$ para el conjunto de Bilbao), un mayor porcentaje de personas ocupadas en profesiones no cualificadas (el 8,2\% frente al 5,4\% en el conjunto del municipio) y una renta personal media, también inferior a la media del municipio ( 10.357 frente a 17.670 de media en Bilbao), los barrios de este grupo exhiben un perfil marcado por la vulnerabilidad y el riesgo a la exclusión. El porcentaje de viviendas en régimen de alquiler es del $14,8 \%$, significativamente superior al de la media del municipio $(9,8 \%)$ ,y el índice de confort de las viviendas de este grupo de barrios es inferior a la media (58,5 frente al 68,7 que corresponde a la media de Bilbao). Se trata de barrios con población poco cualificada y con viviendas de menor confort que la media del municipio.

El grupo 7 está formado por el barrio de San Francisco. Este barrio presenta rasgos específicos derivados de la presencia destacada de población extranjera (32,8\% de la población total) y las precarias condiciones económicas de parte de la población.

Para enfatizar el carácter espacial del análisis realizado, se han trasladado los resultados obtenidos en el Análisis Clúster a un mapa (Figura 5), donde aparecen en diferentes colores los barrios en función del grupo al que pertenecen y donde se puede observar que los resultados estadísticos obtenidos tienen su reflejo en las dinámicas de la ciudad lo que permite una mejor compresión de las estructuras subyacentes.

En el mapa de la Figura 5 se puede observar una estructura caracterizada por un centro y varias periferias o anillos periféricos, donde Abando e Indautxu (Grupo 1) destacan como centro neurálgico y diferenciado del resto de los barrios, tal y como hemos señalado anteriormente. El barrio de San Francisco destaca como un valor atípico o outlier, con características propias, pese a su proximidad geográfica con el grupo compuesto por Abando e Indautxu, con características radicalmente opuestas en las cuatro dimensiones estudiadas y que en la siguiente sección se explicarán en detalle. Rodeando el grupo l se sitúa un primer anillo periférico que está compuesto por los barrios que pertenecen a los grupos 2 y 3 que son barrios predominantemente residenciales con una capacidad socio-económica media-alta. Un segundo anillo suburbano cierra la ciudad por el sur, con un grupo (Grupo 4) compuesto por barrios con unas condiciones socio-económicas inferiores a los integrados en el anillo anterior y que han quedado fuera de las dinámicas económicas y sociales que se desarrollan en el resto de la ciudad. El trazado de la autopista A-8, junto con la ausencia de líneas de metro en toda la zona sur de la ciudad, ha podido contribuir a esta configuración geográfica y socio-económica de estos barrios.

Por último, estarían los barrios incluidos en los grupos 5 y 6, que, si bien 


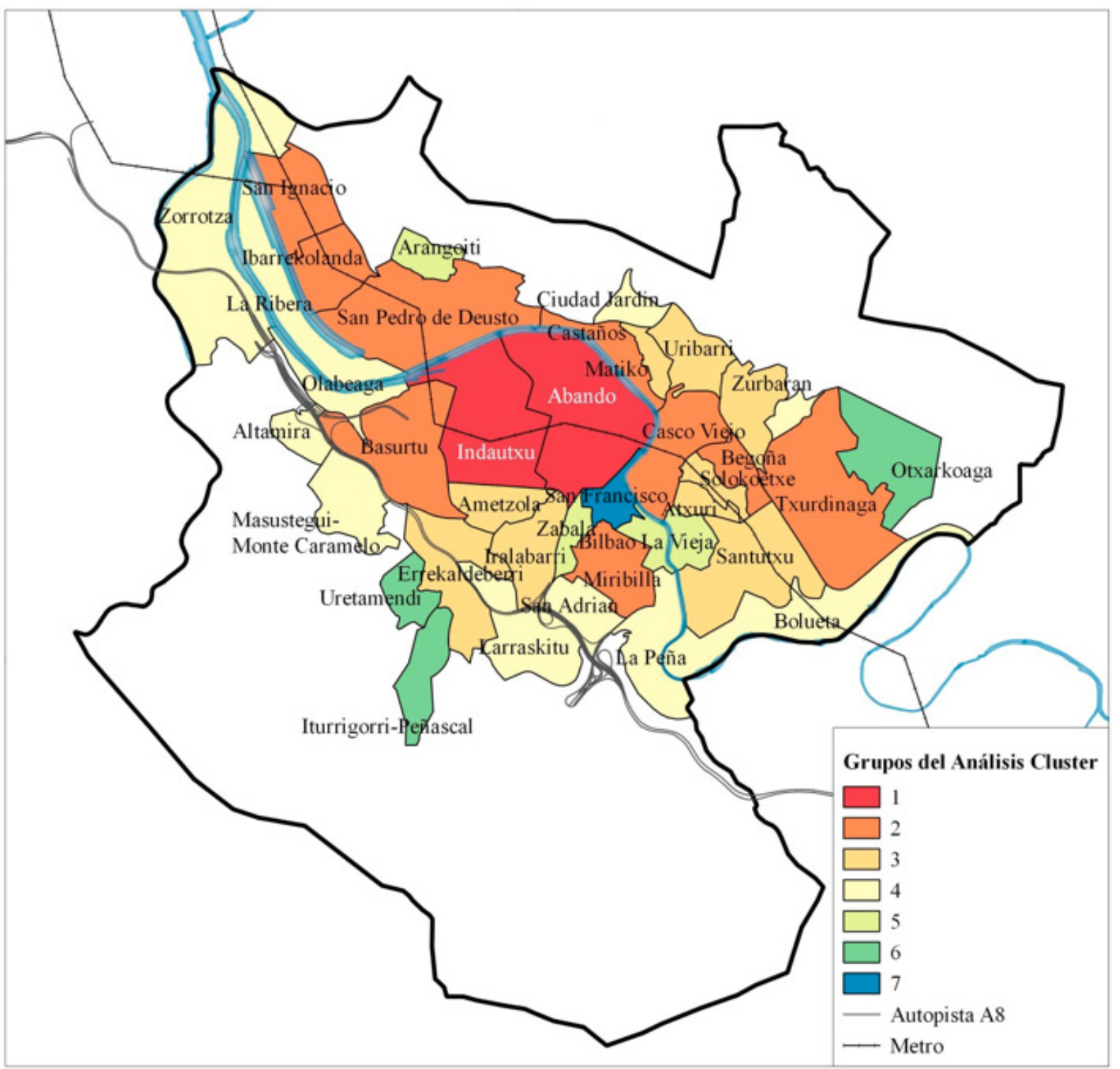

Figura 5: Agrupación Clúster de los barrios de Bilbao

podemos considerar que están integrados en los anillos anteriores, por diversas razones han quedado desconectados de las dinámicas que estamos analizando y que caracteriza a los anillos suburbanos. Entre estas razones podríamos citar la ausencia de rehabilitación del parque de viviendas que ha atraído a población de menos recursos y menos cualificación debido a la contención relativa del precio de las viviendas.

En definitiva, los resultados obtenidos mediante el AFM y el Análisis Clúster y que se han mostrado en un mapa, muestran que la agrupación de los barrios de Bilbao en 7 grupos diferenciados según las variables seleccionadas, reflejan estructuras y dinámicas que se pueden observar actualmente en el municipio, por lo que, podrían, a su vez, servir de base para la aplicación de políticas urbanas más ajustadas a la realidad. 


\section{Representación gráfica/cartográfica de la estratificación socio- espacial de los barrios de Bilbao en cada una de las dimensiones}

En el apartado anterior se ha representado gráficamente la posición media relativa que alcanza cada barrio teniendo en cuenta conjuntamente la información de los cuatro grupos de variables. Sin embargo, una de las virtualidades de la técnica estadística es que permite proyectar en el plano factorial los barrios desde los distintos puntos de vista que se han considerado. Así, cada barrio podría representarse en el plano factorial mediante cuatro puntos, uno de ellos reflejaría la posición media relativa del barrio y, el resto, las posiciones parciales relativas de cada barrio correspondiente a cada uno de los tres puntos de vista. El plano factorial en el que los individuos aparecen representados por diferentes puntos es de gran riqueza interpretativa porque permite detectar la existencia de barrios con comportamientos heterogéneos, en el sentido que la posición que ocupan en un determinado punto de vista no corresponde a la posición que presentan en otro u otros.

En las Figuras 1A-4A (Apéndice) se proyectan los barrios según cada uno de los puntos de vista estudiados. La Figura lA recoge la posición relativa de los barrios en relación con la dimensión demográfica. De acuerdo con el eje de abscisas, los barrios se ordenan en función de la dimensión demográfica de mayor a menor densidad de población ( total y extranjera) y de mayor a menor concentración de población extranjera. El barrio de San Francisco encabeza el ranking, seguido a una distancia significativa del resto de barrios. Santutxu, Ametzola, Indautxu, Uribarri, Zabala, Abando e Iturralde serían también barrios que ocupan una posición destacada. Sin embargo, la posición de estos últimos barrios obedece particularmente al volumen de población que reside en ellos más que a la diversidad de la población ubicada en ellos (como ocurre en San Francisco). Cierran el ranking un grupo de barrios, todos ellos situados en la corona exterior de la ciudad: La Ribera, Ciudad Jardín, Elorrieta, Larraskitu, Altamira, Bolueta, Arabella, Masustegui e Iturrigorri, y todos ellos barrios con un bajo volumen de población, baja densidad de población y poca presencia de población extranjera (cuadrante noreste de la Figura 6). Las diferencias entre los barrios en esta dimensión son muy significativas, lo que se puede apreciar en la amplitud del eje de ordenadas. En el mapa de la Figura 7 se representa el valor de cada barrio según la dimensión demográfica. En él se puede apreciar la distribución espacial de los barrios de acuerdo a la dimensión referida. Se observa que San Francisco presenta los valores más elevados debido a la fuerte presencia de población inmigrante y a la alta densidad poblacional tanto local como extranjera. Los barrios periféricos registran valores más bajos como resultado de una menor densidad demográfica y menor presencia de población inmigrante. 


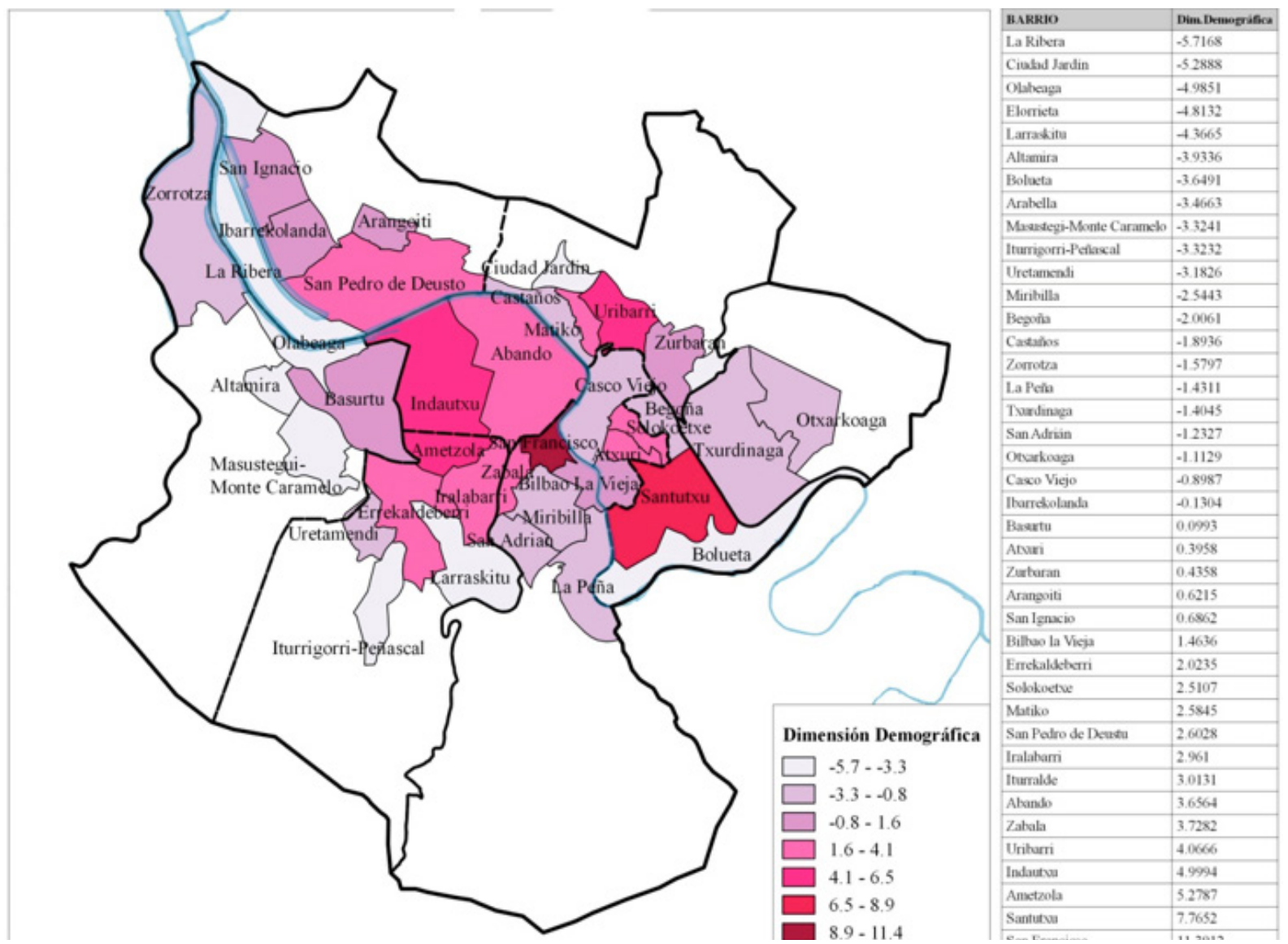

Figura 6: Barrios de Bilbao según la Dimensión Demográfica

Nota: La leyenda muestra las coordenadas del segundo factor (que es el que explica la dimensión demográfica) en diferentes tramos. Los valores positivos más elevados corresponden a barrios con mayor complejidad demográfica.

La Figura 2A (Apéndice) presenta la posición relativa de los barrios en relación con la condición socio-económica. En este caso, se observa que la amplitud del eje de abscisas es mayor que en el anterior, lo que se interpreta como una mayor dispersión entre los barrios. Esto es, las diferencias entre los barrios en relación con la situación socio-económica son mayores que las que existen en las condiciones de demografía. Los barrios que abren este nuevo ranking son Indautxu, Abando, Castaños y Miribilla, todos ellos caracterizados por un nivel de renta y un porcentaje de ocupados en profesiones superiores e intermedias superior a la media. Cierran el ranking los barrios Iturrigorri, Otxarkoaga, San Francisco y Uretamendi, estos, por el contrario, con una renta inferior y con ocupados en profesiones superiores e intermedias también inferior. En el mapa de la Figura 7 se muestra la distribución de los barrios según esta dimensión. Se observa nuevamente una estructura centro-periferia, en la que barrios acomodados como Indautxu, Abando o Castaños se sitúan en el centro y en torno a ellos aparecen dos anillos periféricos caracterizados por una situación socio-económica cada vez menos favorable. Destaca la situación de Miribilla, un barrio de reciente urbanización. 


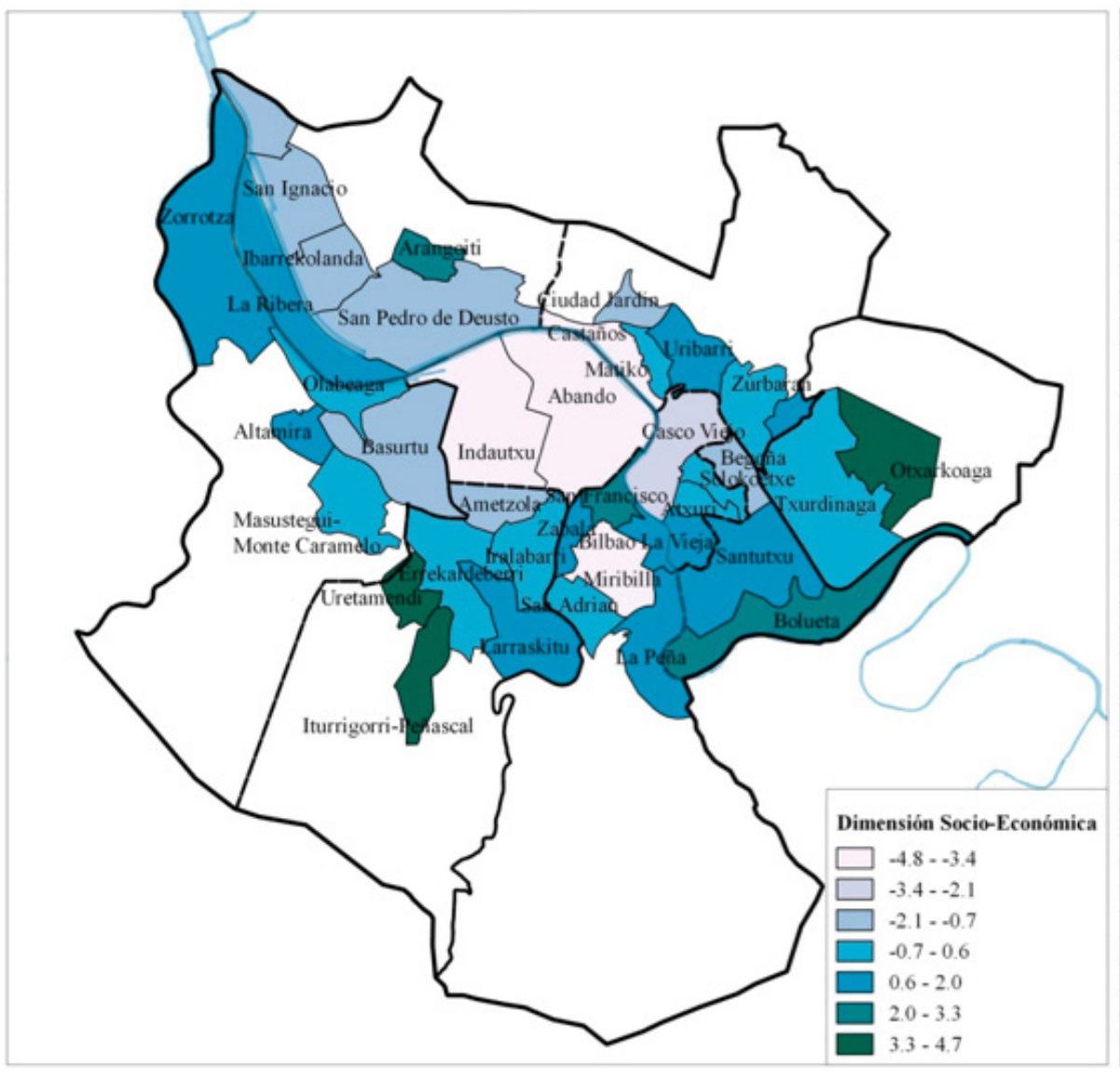

\begin{tabular}{|c|c|}
\hline В Аизао & Dim Sackb Ereanimbira \\
\hline Ahandb & -4.8013 \\
\hline Indutua & -4.6884 \\
\hline Minibilla & .39538 \\
\hline Castaños & .3 .7174 \\
\hline Casoo Vico & -28003 \\
\hline San Padro de Dewatu & -2.0053 \\
\hline Ametrola & -1836 \\
\hline San Igracio & -1.7078 \\
\hline Elomicta & -1.7078 \\
\hline Basutu & .1 .7026 \\
\hline Ciubded Jardin & -1.3605 \\
\hline Begoña & -12258 \\
\hline Pluerdiolanda & -1.0535 \\
\hline Iturralde & -0.5929 \\
\hline Matiko & -0.4304 \\
\hline Solokoetse & -0.272 \\
\hline Masndegi-Monte Caranelo & -0.2094 \\
\hline Txurdingya & 0.2055 \\
\hline Iralakarn & 0.18 \\
\hline Zurturan & 0.1636 \\
\hline Olabeap & 0.1796 \\
\hline Errdaldstemi & 0.3344 \\
\hline San Adriain & 0.4683 \\
\hline Santutua & 0.6293 \\
\hline Atsuri & 0.6504 \\
\hline Uniturni & 0.6913 \\
\hline Bellhwo la Vicja & 0.6981 \\
\hline La Rilera & 1.0024 \\
\hline Altamira & 1.3776 \\
\hline Arabella & 1.3958 \\
\hline Zorrota & 1.6125 \\
\hline La Péna & 1.7416 \\
\hline Larraditu & 1.7528 \\
\hline Zatula & 1926 \\
\hline Arangoiti & 2 \\
\hline Bolucta & 20024 \\
\hline San Franciso & 3.2696 \\
\hline Unetamenali & $3 \times 262$ \\
\hline Otsaktouga & 43369 \\
\hline Iturrigorri-Pehuscal & 4.6782 \\
\hline
\end{tabular}

Figura 7: Barrios de Bilbao según la Condición Socio-económica

Nota: La leyenda muestra las coordenadas del primer factor (que es el que explica la socio-económica) en diferentes tramos. Los valores positivos más elevados corresponden a barrios con mejor posición socio-económica.

En la Figura 3A (Apéndice) se proyecta la posición relativa de los barrios en relación con las características de la vivienda. En este caso, los barrios se ordenan a lo largo del eje de abscisas (de izquierda a derecha) de mayor a menor índice de confort, superficie de la vivienda por persona y porcentaje de viviendas en régimen de propiedad. Los barrios de Abando e Indautxu ocupan las primeras posiciones por ser barrios con una tipología de vivienda de calidad (edificios del ensanche bilbaíno, con viviendas amplias de buena construcción) y una superficie media útil por individuo significativamente superior a la media y un índice de confort más elevado. El barrio de Miribilla aparece en tercer lugar. Esa posición está relacionada con su condición de barrio de reciente creación, con una antigüedad media de las viviendas de 7,7 años. Igualmente, el índice de confort y el tamaño de las viviendas son superiores a la media. El 83\% de las viviendas están régimen de propiedad, aunque sobre el $60 \%$ del total pende una hipoteca. El ranking en base a la dimensión relativa a las características de la vivienda se cierra con Uretamendi, San Francisco, Otxarkoaga e Iturrigorri-Peñascal donde las viviendas tienen bajos índices de confort, tamaño pequeño y se habitan 
en un porcentaje superior a la media en régimen de alquiler. Las diferencias entre los barrios en esta dimensión, aunque importantes, son menores que las que se observaban en la dimensión demográfica. Los resultados se muestran en el mapa de la Figura 8. Se observa que este mapa tiene algunas coincidencias con el anterior (por ejemplo, la situación de Miribilla, Indautxu, Castaños, Abando, etc.) ya que la correlación entre la situación socio-económica y las características de la vivienda es muy elevada.

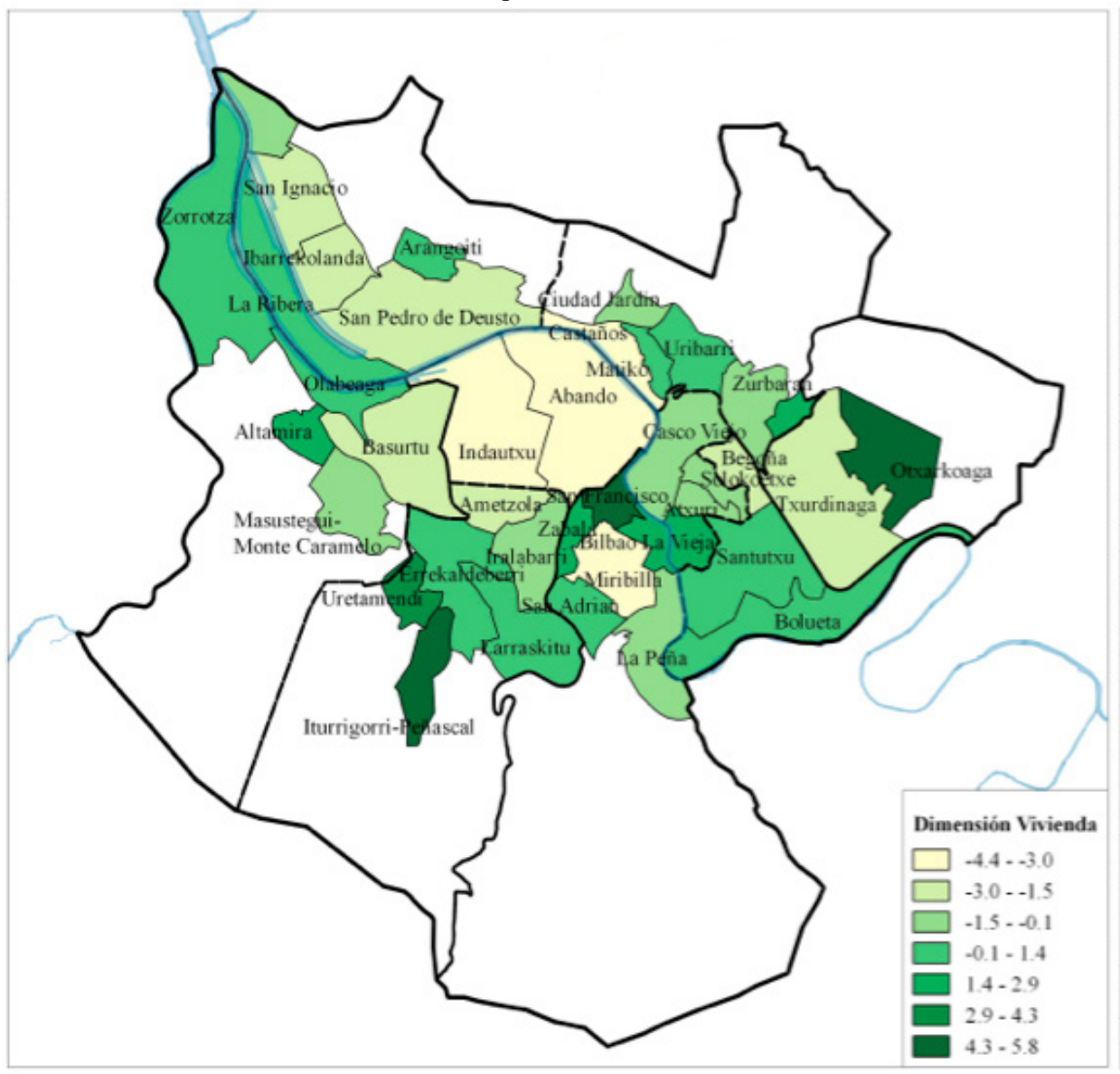

\begin{tabular}{|c|c|}
\hline BURRIO & Dan Vhiends. \\
\hline Miribtlla & $-4+402$ \\
\hline Alanab & -36179 \\
\hline Catafiess & -35419 \\
\hline ladretsu & -3.421 \\
\hline Begola & -26572 \\
\hline thurrekolanda & -19879 \\
\hline Basurte & -19647 \\
\hline Tvudiang & -1.7337 \\
\hline Sun Ignacio & -16877 \\
\hline Anntrola & -16737 \\
\hline San Pelro de Deasu & -1.646 \\
\hline Zurturan & -11664 \\
\hline Cindad Jaxfin & -09935 \\
\hline Elornicta & -08811 \\
\hline Casoo Vigo & -07834 \\
\hline Solvkostre & -06311 \\
\hline Mnevacgi-Monte Caranedo & -05569 \\
\hline La Perì & -204 \\
\hline Inalshen & -0.1847 \\
\hline Itumalde & -0.163 \\
\hline Suntavu & -00195 \\
\hline La Ribera & 0.0213 \\
\hline San Mdrín & 0.0393 \\
\hline Matike & 0.0899 \\
\hline Olateaga & 0.1636 \\
\hline Unibarn & 0.2471 \\
\hline Emdakleberri & 02503 \\
\hline Zonota & 0.4952 \\
\hline Larraditu & 0.713 \\
\hline Arargeviti & 1.2029 \\
\hline Bolueta & 1.3062 \\
\hline Atcari & 1.8335 \\
\hline Zatoba & 1.4999 \\
\hline Arabella & 1.8742 \\
\hline Billhoo b Vicja & 2.1584 \\
\hline Altamina & 2.2633 \\
\hline thetamenh & 42761 \\
\hline San Francisco & 4.4958 \\
\hline Otvarkoapa & 48792 \\
\hline Iturngempletiaxal & 5.785 \\
\hline
\end{tabular}

Figura 8: Barrios de Bilbao según las Características de la Vivienda

Nota: La leyenda muestra las coordenadas del primer factor (que es el que explica las características de la vivienda) en diferentes tramos. Los valores positivos más elevados corresponden a barrios con mejores características.

Por último, la Figura 4A (Apéndice) proyecta la posición relativa de los barrios con respecto a la cohesión social. Los barrios de Iturrigorri-Peñascal, San Francisco, Bilbao La Vieja, Otxarkoaga y Uretamendi encabezan este ranking (de derecha a izquierda) de menor a mayor. La posición de barrios se asocia a un porcentaje relativamente alto de población no cualificada y el mayor peso de viviendas en régimen de alquiler. En el lado opuesto del ranking se encontrarían Abando, Indautxu, Begoña, Ciudad Jardín y Castaños, donde se da un menor porcentaje de población no cualificada y un menor porcentaje de viviendas en régimen de alquiler. Los resultados se pueden ver en la Figura 9, en el que se observa un centro que se amplía 
hacia el noroeste de la ciudad y crea al sureste dos islotes (Miribilla y Begoña), todos ellos caracterizados por una fuerte cohesión social.

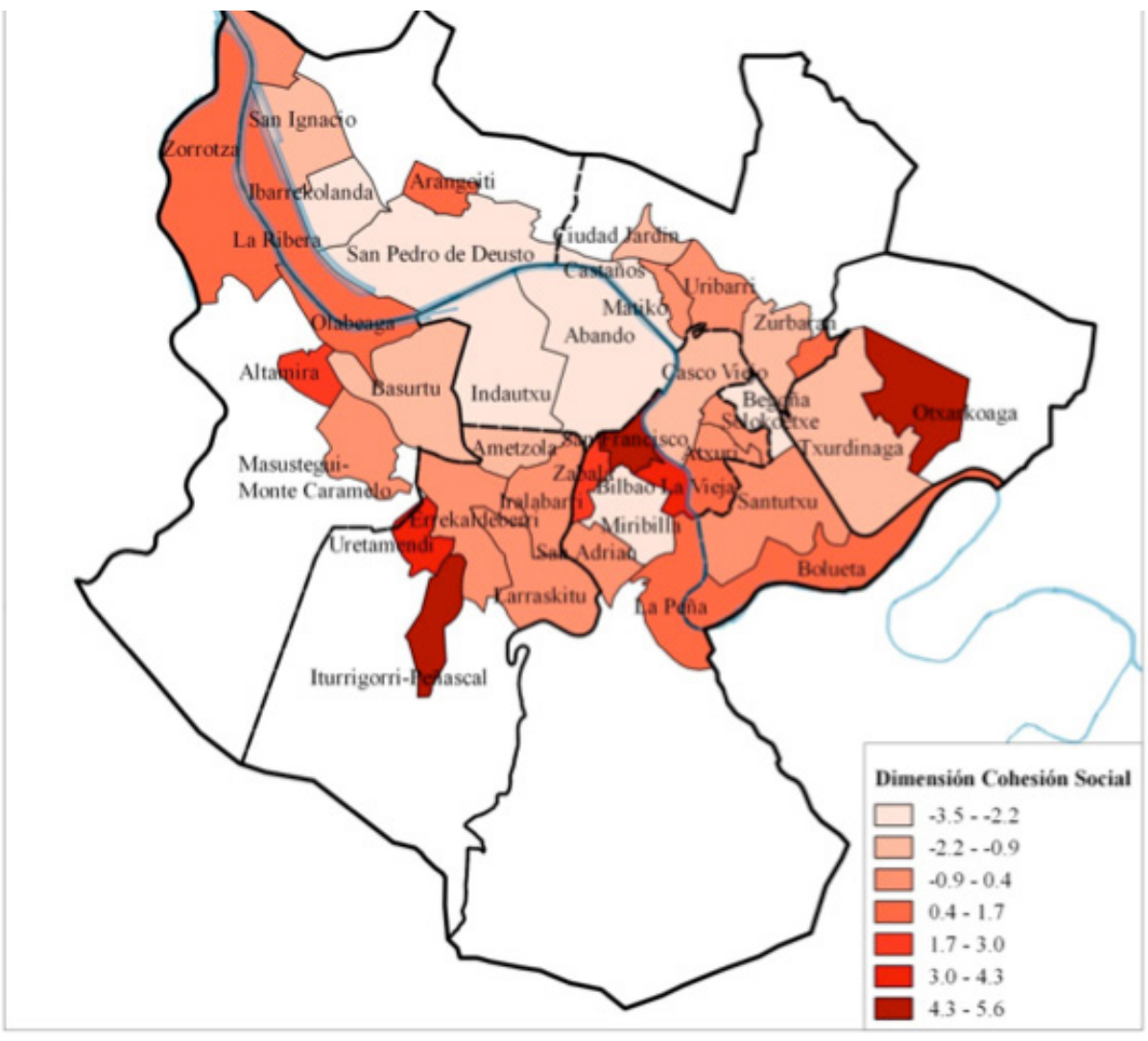

\begin{tabular}{|c|c|}
\hline Begona & -2.74 \\
\hline Aluabs & -2.726 \\
\hline San Pollo de Deusu & $-23 \times 28$ \\
\hline Minbilla & -22621 \\
\hline Pherrdolanda & -22455 \\
\hline Basutu & -2.1764 \\
\hline Cindud Jantin & -2.1176 \\
\hline Amotroba & -20163 \\
\hline San Ignxio & -1.3684 \\
\hline Zutturan & -1.2048 \\
\hline Tardingega & -1.1839 \\
\hline Caso Viejo & -1.1377 \\
\hline Solokiestve & -08103 \\
\hline Matike & -0.7974 \\
\hline Elomixta & -06336 \\
\hline Santitum & -05168 \\
\hline San Adrin & -105032 \\
\hline Itumalle & -0.3223 \\
\hline Inalakam & -02985 \\
\hline Untuen & -0215 \\
\hline Mandsy-Muede Caramelo & -0.109 \\
\hline Lamaditu & 0.187 \\
\hline Endaluktemin & 0.3307 \\
\hline Otaheap: & 0.446 \\
\hline Zenotza & $0.47 m$ \\
\hline Boflust & 0.5627 \\
\hline La Ribera & 0.8096 \\
\hline La Feña & 0.8275 \\
\hline Atsui & 0.921 \\
\hline Arabella & 1.1388 \\
\hline Arangoiti & 1.36 \\
\hline Altamira & 1.7512 \\
\hline Zstuh & 2.7131 \\
\hline Unetameadi & 3.5741 \\
\hline Balhao la Vieja & 3.9924 \\
\hline Otcaritouga & 49441 \\
\hline IturrigomiPlahecal & 5.1135 \\
\hline San Franciseo & 5.5688 \\
\hline
\end{tabular}

Figura 9: Barrios de Bilbao según la Cohesión Social

Nota: La leyenda muestra las coordenadas del primer factor (que es el que explica la cohesión social) en diferentes tramos. Los valores positivos más elevados corresponden a barrios con mejores características.

Como se ha presentado en el epígrafe anterior, en algunos casos el análisis clúster realizado clasifica en un único grupo barrios que pertenecen a un mismo distrito. Este es el caso del distrito 6, compuesto por Abando e Indautxu y también se puede observar cierta correspondencia entre los barrios que componen en grupo-clúster 5 y los del distrito 5. En los demás casos, los barrios que pertenecen a un mismo distrito administrativo muestran realidades muy dispares y se agrupan en clústeres diferentes, lo que pone de manifiesto que la proximidad o contigüidad de unos barrios a otros no explica de manera adecuada las características de los espacios y que es necesario introducir otros parámetros y técnicas de análisis de las unidades territoriales urbanas. El análisis realizado permite, asimismo, identificar cuáles son los principales puntos en común y divergencias dentro de los grupos de barrios resultantes (ver Cuadro 3) para las dimensiones consideradas. Así, aunque se trata de resaltar las pautas comunes, resulta igualmente relevante poder visibilizar las dimensiones menos compartidas dentro de cada grupo de clúster resultante. 
Cuadro 3: Correspondencia entre los barrios de los distritos administrativos y los grupos-clúster y heterogeneidad según las dimensiones del análisis factorial

\begin{tabular}{|c|c|c|c|c|c|c|}
\hline Grupo-Cluster & $\begin{array}{c}\text { Distrito } \\
\text { administrativo }\end{array}$ & Barrio & Demografía & $\begin{array}{l}\text { Condición } \\
\text { socio-económica }\end{array}$ & Vivienda & Cohesión social \\
\hline \multirow{2}{*}{ Grupo 1} & 6 & Abando & ++ & ++ & ++ & ++ \\
\hline & 6 & Indautxu & ++ & ++ & ++ & ++ \\
\hline \multirow{9}{*}{ Grupo 2} & 8 & Basurtu & + & ++ & ++ & ++ \\
\hline & 4 & Begoña & - & + & ++ & ++ \\
\hline & 5 & Casco Viejo & - & ++ & + & + \\
\hline & 2 & Castaños & - & ++ & ++ & ++ \\
\hline & 1 & Ibarrekolanda & + & + & ++ & ++ \\
\hline & 5 & Miribilla & - & ++ & ++ & ++ \\
\hline & 1 & San Ignacio & + & ++ & ++ & + \\
\hline & 1 & $\begin{array}{l}\text { San Pedro Deust } \\
\text { Deustu Deustu }\end{array}$ & ++ & ++ & + & ++ \\
\hline & 3 & Txurdinaga & - & + & ++ & + \\
\hline \multirow{9}{*}{ Grupo 3} & 7 & Ametzola & ++ & ++ & ++ & ++ \\
\hline & 7 & Errekaldeberri & + & - & - & - \\
\hline & 7 & Iralabarri & ++ & + & + & - \\
\hline & 5 & Iturralde & ++ & + & + & + \\
\hline & 2 & Matiko & + & + & - & + \\
\hline & 4 & Santutxu & ++ & - & - & + \\
\hline & 5 & Solokoetxe & + & + & + & + \\
\hline & 2 & Uribarri & ++ & - & - & - \\
\hline & 2 & Zurbaran & + & + & + & + \\
\hline \multirow{12}{*}{ Grupo 4} & 2 & Ciudad Jardín & - & + & + & ++ \\
\hline & 8 & Altamira & - & - & -- & - \\
\hline & 2 & Arabella & -- & - & -- & -- \\
\hline & 4 & Bolueta & - & - & -- & - \\
\hline & 1 & Elorrieta & -- & ++ & + & + \\
\hline & 5 & La Peña & - & -- & + & - \\
\hline & 1 & La Ribera & - & - & - & - \\
\hline & 7 & Larraskitu & - & -- & - & - \\
\hline & 8 & $\begin{array}{l}\text { Masustegi-Monte } \\
\text { Caramelo }\end{array}$ & -- & + & + & - \\
\hline & 8 & Olabeaga & -- & - & - & - \\
\hline & 5 & San Adrián & - & - & - & + \\
\hline & 8 & Zorrotza & - & -- & - & - \\
\hline \multirow{4}{*}{ Grupo 5} & 1 & Arangoiti & + & -- & - & - \\
\hline & 5 & Atxuri & + & - & - & - \\
\hline & 5 & Bilbao la Vieja & + & - & -- & - \\
\hline & 5 & Zabala & ++ & -- & - & - \\
\hline \multirow{3}{*}{ Grupo 6} & 7 & Iturrigorri-Peñascal & - & -- & - & - \\
\hline & 3 & Otxarkoaga & - & -- & - & -- \\
\hline & 7 & Uretamendi & - & $-\cdot$ & -- & - \\
\hline Grupo 7 & 5 & San Francisco & ++ & -- & -- & -- \\
\hline
\end{tabular}

Fuente: Elaboración Propia 
Nota 1: En este cuadro se ha señalado en color verde oscuro los barrios que ocupan las primeras diez posiciones en la dimensión correspondiente; en color verde claro los barrios que ocupan las siguientes diez posiciones; en color naranja claro, las posiciones 21 a la 30 y, por último, en naranja oscuro aparecen los barrios que ocupan las últimas posiciones para cada dimensión.

Nota 2: En la primera de las dimensiones (demografía) se señalan en verde oscuro los barrios con mayor población/densidad de población local y extranjera.

La aplicación del análisis AFM y Cluster al caso de Bilbao revela así un mapa sintético de la estratificación urbana donde los barrios de Abando e Indautxu (Grupo 1) constituyen el lugar central de Bilbao y se sitúan en lo más alto de la jerarquía socio-económica de la ciudad; estos barrios comparten una posición muy ventajosa en todas y cada una de las dimensiones estudiadas. Los barrios del Grupo 2 comparten principalmente unas buenas condiciones socio-económicas, de vivienda y de cohesión y, aunque en el ámbito de demografía no se trata en general de barrios especialmente populosos, existen casos con elevada densidad poblacional entre ellos (San Pedro de Deustu). Por su parte, el Grupo 3 está formado por barrios de alta densidad de población y se aprecian en general situaciones intermedias en las dimensiones de condición socio-económica, vivienda y cohesión social. Destaca por su buen posicionamiento en estas tres áreas el barrio de Ametzola, que sería el mejor situado dentro de este grupo, aunque no llega a los umbrales necesarios para ser considerado dentro de los grupos anteriores. A continuación, dentro del cuarto grupo se encuentran barrios que comparten una baja densidad poblacional y condiciones medias-bajas en términos de condición socio-económica, características de la vivienda y cohesión social. Dentro de este grupo se encuentra relativamente mejor situado el barrio de Ciudad Jardín, aunque se trata de un barrio con escasa representación poblacional en el conjunto de la ciudad. Elorrieta también destaca por su relativa buena posición en la dimensión socio-económica.

Los tres últimos grupos presentan los peores indicadores en términos laborales, de renta, de calidad de vivienda y tasa del paro, aunque con importantes diferencias entre ellos. Así, los barrios que integran el quinto grupo tienen una media-alta densidad de población y, a excepción de Arangoiti, están situados en áreas centrales de la ciudad. Asimismo, los procesos de gentrificación en curso contribuyen a explicar los mejores resultados relativos en la dimensión socio-económica del barrio de Bilbao La Vieja.

Iturrigorri, Otxarkoaga y Uretamendi conforman el Grupo 6 y forman parte de la periferia de la ciudad en términos espaciales, socio-económicos y urbanísticos. Así, unida a su baja densidad poblacional se concentran en ellos bajos niveles de ocupación, de renta y de calidad de la vivienda. Aunque no comparten un mismo distrito administrativo, tal y como se aprecia por los resultados constituyen un ejemplo claro de barrios vulnerables.

Por último, el barrio de San Francisco (Grupo 7) presenta rasgos específicos derivados principalmente de las precarias condiciones económicas de la población, por la alta densidad de población extranjera y por el grave deterioro de las condi- 
ciones de la vivienda y el hábitat. Estas características sitúan este barrio dentro de la tipología clásica de barrios desfavorecidos, vulnerables o en crisis, situados en las zonas centrales de las ciudades.

En resumen, el análisis de la agrupación de los barrios en función del conjunto de las variables se puede enriquecer haciendo agrupaciones alternativas en función de las diferentes dimensiones que se quiera analizar. La metodología presentada permite un análisis más afinado de la problemática de los barrios (u otras escalas) y, por tanto, la posibilidad de definir políticas públicas más ajustadas a esas problemáticas específicas en el territorio definido.

\section{Conclusiones}

Las tendencias hacia una creciente tercerización de la economía, profesionalización y desregulación de los mercados de trabajo junto con el debilitamiento del estado social y ensanchamiento de la desigualdad en el reparto de la renta y la riqueza, en las economías industriales avanzadas, no han hecho sino agudizarse durante la crisis financiera y económica que comienza en el año 2007, una tendencia a la que han contribuido de manera activa y contundente las políticas austeritarias impulsadas por los gobiernos en la mayoría de los países. El estallido de la crisis abocó los sectores productivos tradicionales a una fuerte reestructuración mientras que sectores como el financiero o los servicios a las empresas han mantenido su protagonismo en la economía. Al mismo tiempo, la prioridad del control del gasto público ha impuesto la austeridad como condición y alentado la puesta en marcha de reformas y ajustes extraordinarios en las distintas partidas que conforman la base del Estado de Bienestar y que han traído como consecuencia un ahondamiento en la precariedad y desprotección de capas cada vez más amplias de la población. Unido a todo ello, los programas de ajuste implementados y las reformas laborales acometidas y dirigidas a su flexibilización han desembocado en una creciente precarización de las condiciones de trabajo generales que han afectado en mayor medida a las sectores sociales más vulnerables. El resultado de estos procesos ha desembocado en un aumento de las desigualdades sociales que han profundizado si cabe en la ya de por sí amplia brecha existente antes del estallido de la crisis.

En general, los procesos identificados han tenido una clara impronta territorial, que ha evidenciado no sólo el peso y jerarquización relativa de unos espacios frente a otros, sino, también, en las nuevas reconfiguraciones socio-espaciales en su interior. Así, se asiste al surgimiento de ciudades y metrópolis que ocupan los primeros puestos en los rankings internacionales, nuevos sectores de actividad dinámicos, grupos profesionales de éxito y zonas dentro de las ciudades que reúnen los requisitos para ser promocionadas como centro neurálgico de la vida económica y cultural de las principales urbes. Sin embargo, paralelamente a estas tendencias, son numerosos los estudios que evidencian la reconfiguración de nuevas realidades que van alejándose de este foco y se van ubicando en un espacio crecientemente desligado de estas 
dinámicas exitosas. De manera que el impacto de la crisis financiera y económica se hace notar con especial intensidad en determinados barrios y áreas urbanas como consecuencia de la segregación espacial de los sectores sociales más castigados que hace que la problemática social más aguda se concentre en esos espacios.

En este contexto, resulta de especial interés identificar los elementos diferenciales relacionados no sólo con los mecanismos de exclusión social sino también con dinámicas de expulsión que no estarían vinculadas con las tradicionales características socio-económicas sino con otras más específicas y asociadas a las mejoras en el bienestar de las personas, entendido este en un sentido amplio que incluye también la posibilidad de participar en los procesos de decisión dentro de la comunidad. Las técnicas estadísticas multivariante son un instrumento de gran potencial para avanzar en el análisis conjunto de estas tres dimensiones en términos de la diferenciación y estratificación socio-espacial y de especial relevancia para la intervención pública en la ciudad.

En el caso de Bilbao, la metodología basada en el Análisis Factorial Múltiple (AFM) permite una caracterización de los barrios de Bilbao descrita por variables cuantitativas y define las posiciones relativas de los barrios de la ciudad en relación con variables demográficas, urbanísticas, socio-económicas y de cohesión social revelando un Mapa Sintético de la Estratificación Socio-Espacial de la ciudad. A partir de estos resultados, el Análisis Clúster permite agrupar a los 40 barrios de la ciudad en siete grupos o clases diferentes que comparten características homogéneas en cuanto a sus rasgos demográficos, urbanísticos, socio-económicos y de cohesión social. La clasificación obtenida permite un análisis en profundidad de la estratificación urbana en base a un conjunto de variables ponderadas. Ese conocimiento de la estructura urbana subyacente hace posible identificar realidades compartidas por ámbitos territoriales que no forman parte de las mismas unidades administrativas ni están en situación de proximidad física, pero que son homogéneas desde el punto de vista de una problemática compartida por variables agrupadas que se comportan de manera similar. Además, permite observar con nitidez la realidad de barrios situados en posiciones intermedias que, de otra manera, son difíciles de caracterizar en base a métodos univariantes tradicionales. No obstante, se trata de una "fotografía" fija de la ciudad en un momento determinado (año 2011), que requiere de un mayor desarrollo para poder avanzar en un análisis dinámico y así poder identificar la evolución de las dinámicas de segregación socio-espacial en la ciudad en diferentes periodos de tiempo.

A partir de la aplicación de la metodología descrita es posible obtener una clasificación alternativa de los barrios de Bilbao superando los límites administrativos y de proximidad tradicionales, que permite una perspectiva transversal en la formulación y aplicación de las políticas urbanas en cada una de las dimensiones estudiadas. La nueva clasificación que se obtiene a partir de la aplicación del Análisis Factorial Múltiple y de Cluster agrupa los barrios en función del comportamiento ponderado de un conjunto de variables socio-económicas, demográficas y urbanísticas. Las agrupaciones reflejan las estructuras subyacentes en la dinámica socio-espacial 
urbana perfilando un mapa sintético de la estratificación de los barrios de Bilbao. De este modo, se obtienen nuevas agrupaciones que mejoran el conocimiento de las estructuras subyacentes y la estratificación de los barrios en grupos homogéneos lo que permite definir intervenciones más ajustadas a las especificidades de cada conjunto de barrios.

En resumen, la utilización de técnicas estadísticas multivariante permite analizar en profundidad y en distintas dimensiones las estructuras urbanas teniendo en cuenta las posiciones relativas de los barrios y sus poblaciones en función de características demográficas, socio-económicas o urbanísticas y, por tanto, identificar con mayor precisión las múltiples facetas en las que se traducen las dinámicas territoriales. En este sentido, constituye una importante herramienta para avanzar en el conocimiento de los procesos de segregación socio-espacial y sus dimensiones, realizar comparaciones entre diferentes lugares y también, en distintos momentos. En definitiva, este análisis permite ir más allá de una visión dicotómica de la estructura socio-espacial urbana centrada en los barrios tradicionales y los nuevos; los del centro y la periferia; los más ricos y los más pobres; y pasar a un análisis multidimensional que enriquezca el análisis, la caracterización, mostrando las diferentes realidades superpuestas en el entramado urbano. Con la metodología desarrollada en este trabajo, la clásica distribución de los barrios en torno a los distritos urbanos en los que la proximidad era una de los elementos utilizados para su clasificación, deja paso a agrupaciones más versátiles, dinámicas y fructíferas de barrios que podrían eventualmente actuar como interlocutores en el diseño de la política urbana para cada uno de los ámbitos de actuación.

\section{Bibliografía}

ABASCAL, E., AGUIRRE, K. y LANDALUCE, M. Técnicas factoriales de análisis de tablas múltiples. Nuevos desarrollos empíricos. Universidad del País Vasco-Euskal Herriko Unibertsitatea, 2001, p. 1-27.

ALGUACIL, J., CAMACHO, J. y HERNÁNDEZ, A. La vulnerabilidad urbana en España. Identificación y evolución de los barrios vulnerables. Empiria. Revista de metodología de ciencias sociales, 2014, 27, p. 73-94.

ANTOLÍN, E., FERNÁNDEZ, J. M. y LORENTE, E. Estrategias de regeneración urbana y segregación residencial en Bilbao: apariencias y realidades. Ciudad y Territorio: Estudios Territoriales, 2010, n ${ }^{\circ}$ 163, p. 67-81.

ARIAS, F. (coord.) La desigualdad urbana en España. Madrid: Ministerio de Fomento, 2000.

BUZAI, G. D. y BAXENDALE, C. A. Aportes del análisis geográfico con Sistemas de Información Geográfica como herramienta teórica, metodológica y tecnológica para la práctica del ordenamiento territorial, Persona y sociedad, 2016, n 27(2), p. 113-141.

BUZAI, G.D. Los mapas sociales urbanos. Buenos Aires: Lugar Editorial, 2003. 
CARAVACA, I., GONZÁLEZ-ROMERO, G., y LÓPEZ, P. Crisis y empleo en las ciudades españolas. EURE (Santiago), 2017, vol. 43, nº128, p. 31-54.

CASTELLS, M. Globalisation, Networking, Urbanisation: Reflections on the Spatial Dynamics of the Information Age. Urban Studies 2010, vol. 47(13) 2737-2745

DE LAS RIVAS, J. L.; FERNÁNDEZ-MAROTO, M.; RODRIGO, E. y MARTÍNEZ, M. Recuperando el concepto urbanístico de barrio: unidades urbanas y regeneración urbana en Castilla y León, Ciudad y Territorio: Estudios Territoriales, 2017, nº191, pp. 45-62.

DEL PINO, J. A. La estructura social urbana de Málaga. Acercamiento a la estructura social urbana a partir de los datos censales sobre condición socio-económica. Cuadernos de Ciencias Económicas y Empresariales, 2001, nº 41, p. 139-162.

DÍAZ MUÑOZ, M.A. Alcalá de Henares: la diferenciación residencial en una ciudad histórica del área metropolitana de Madrid, Anales de geografía de la Universidad Complutense, 1986, vol. 6, p. 253.

DÍAZ PARRA, I. Cambios en la geografía social de Sevilla 1981-2001, Cuadernos Geográficos,2010, nº 46, pp. 139-161.

DONAT, C.; NEL.LO, O. y JIMÉNEZ, E. Crisis, desigualdad social y segregación urbana en Cataluña, paper presentado en GIGAPP-IUIOG 1 de octubre de 2014.

ESCOFIER, B. y PAGÈS, J. Analyses factorielles simples et multiples. Paris: Dunod, 1990.

ESCOFIER, B. y PAGÈS, J. Análisis Factoriales simples y múltiples: Objetivos, Métodos e interpretación. Bilbao: Servicio Editorial de la Universidad del País Vasco, 1992.

ESCOFIER, B. y PAGÈS, J. Multiple factor analysis (AFMULT package). Computational Statistics and Data Analysis, 1994, n 18, p. 121-140.

ESCOUFIER, Y. Le traitement des variables vectorielles. Biometrics, 1973, n 29, p. $751-760$.

ESTEBAN, M. S. Bilbao, luces y sombras del titanio: el proceso de regeneración del Bilbao metropolitano. Bilbao: Universidad del País Vasco- Servicio Editorial, 2000.

FAINSTEIN, S. y HARLOE, M. (ed.). Divided cities: New York \& London in the contemporary world. Oxford: Blackwell, 1992.

FRIEDMANN, J. The World City Hypothesis. Development and Change, 1996, vol. 17, $\mathrm{n}^{0} 1$, p. 69-83.

GARCÍA, I. y ABASCAL, E. Una metodología para el estudio de la evolución de variables latentes. Análisis de las infraestructuras de carreteras de las comunidades autónomas (1975-2000). Estadística Española, 2003, 45 (153), p. 193-210.

GÓMEZ, M. V. Reflective images: the case of urban regeneration in Glasgow and Bilbao. International journal of urban and regional research, 1998, 22(1), p. 106-121.

GUEVARA, T. Abordajes teóricos sobre las transformaciones sociales, económicas y territoriales en las ciudades latinoamericanas contemporáneas. EURE (Santiago), 2015, vol. 41, no 124, 5-24. <https://dx.doi.org/10.4067/S0250-71612015000400001>. [17 de Enero de 2018].

HAMNETT, C. Social polarisation in global cities: theory and evidence. Urban 
studies, 1994, vol. 31, nª 3, p. 401-424.

HARVEY, D. Social justice and the city. Oxford: Blackwell, 1973.

JOSSE, J., PAGÈS, J. y HUSSON, F. Testing the significance of the RV coefficient. Computational Statistics and Data Analysis, 2008, n 53, p. 82-91.

KAISER, H. F. The Application of Electronic Computers to Factor Analysis. Educational and Psychological Measurement, 1960, n 10, p. 141-151.

LEAL, J. y DOMÍNGUEZ, D. Transformaciones económicas y segregación social en Madrid. Ciudad y territorio. Estudios territoriales, 2008, nº 158, 703-726.

LEAL, J. y SORANDO, D. Economic crisis, social change and segregation processes in Madrid. Socio-Economic Segregation in European Capital Cities: East Meets West, 2016, p. 214-237.

LEBART, L. MORINEAU, A. Y PIRON M. Statistique Exploratoire Multidimensionnelle. París: Dunod, 1995,

MADANIPOUR, A., CARS, G. y ALLEN, J. Social exclusion in European cities: processes, experiences and responses. London: Jessica Kingsley, 1998.

MARCOS, M. y MERA, G. La dimensión espacial de las diferencias sociales. Alcances y limitaciones metodológico-conceptuales de las herramientas estadísticas para abordar la distribución espacial intraurbana. Revista Universitaria de Geografía, $2011, \mathrm{n}^{0} 20$, p. 41-65.

MARCUSE, P. y VAN KEMPEN, R. Globalizing cities: A new spatial order?, Oxford: Blackwell, 2000.

MARCUSE, P. "Dual city": a muddy metaphor for a quartered city. International Journal of Urban and Regional Research, vol. 13, 4, 1989, p. 697-708.

MÉNDEZ, R. y PRADA, J. (2014): Crisis, desempleo y vulnerabilidad en Madrid. Scripta Nova. Revista electrónica de Geografía y Ciencias Sociales. [En línea] Barcelona: Universidad de Barcelona, 20 de Abril de 2014, Vol. 18, nº.474. < $\underline{\text { http://www.ub.es/ }}$ geocrit/sn/sn-474.htm>. ISSN: 1138-9788.

MÉNDEZ, R., ABAD, L. D. y ECHAVES, C. Atlas de la crisis. Impactos socioeconómicos y territorios vulnerables en España. Valencia: Tirant Humanidades. 2015.

MINGIONE, E. Urban poverty in the advanced industrial world: concepts, analysis and debates. En E. MINGIONE, Urban poverty and the underclass. Oxford: Blackwell, 1996.

MOULAERT, F., RODRÍGUEZ, A. y SWYNGEDOUW, E. The Globalized City. Economic Restructuring and Social Polarization in European Cities. Oxford: Oxford University Press, 2003.

MOLLENKOPF, J. y CASTELLS, M. Dual City: Restructuring New York. New York: Russell Sage Foundation, 1991.

MUSTERD, S., MARCIŃCZAK, S., VAN HAM, M., Y TAMMARU, T. Socioeconomic segregation in European capital cities. Increasing separation between poor and rich. Urban Geography, 2017, vol. 38, nº 7, p. 1062-1083.

OECD Perspectives on Global Development 2012: Social Cohesion in a Shifting World. Paris : OECD Development Centre, 2011. https://www.oecd-ilibrary.org/develop- 
ment/perspectives-on-global-development-2012_persp_glob_dev-2012-en

PACIONE, M. Britain's cities: geographies of division in urban Britain. London: Routledge, 1997.

PAGÈS, J. Multiple factor analysis. En A. MORINEAU, A. y FERNANDEZ, K. (Eds.) Analyses Multidimensionnelles des données, ANGUS 97», CISIA-CERESTA, 1998, p. 38-48.

PIKETTY, T. El capital en el siglo XXI. Madrid: Fondo de cultura económica, 2014.

PLAZA, B. Evaluating the influence of a large cultural artifact in the attraction of tourism: the Guggenheim Museum Bilbao case. Urban Affairs Review, 2000, 36(2), p. 264-274.

PRATSCHKE, J., y MORLICCHIO, E. Social polarisation, the labour market and economic restructuring in Europe: An urban perspective, Urban Studies, 2012, 49(9), p. 1891-1907.

REQUES, P. Transformaciones espaciales y procesos sociodemográficos en la ciudad. En PUJADAS et al. Población y Espacios Urbanos. Ed. AGE and UB, 2011, p. 235-284.

RODRÍGUEZ, A. MOULAERT, F. y SWYNGEDOUW, E. Nuevas Políticas Urbanas para la revitalización de las ciudades en Europa, Ciudad y Territorio: Estudios Territoriales, 2001, (XXXIII) 129, p. 409-424.

RODRÍGUEZ, A. y MARTÍNEZ, E. Del Declive a la Revitalización: Oportunidades y Límites de las Nuevas Políticas Urbanas en Bilbao, Ciudad y Territorio: Estudios Territoriales, 2001, (XXXIII) 129, p. 441-459.

RODRíGUEZ, A., MARTíNEZ, E. y GUENAGA, G. Uneven redevelopment: new urban policies and socio-spatial fragmentation in metropolitan Bilbao. European Urban and Regional Studies, 2001, 8(2), p. 161-178.

RUBIALES PÉREZ, M.; BAYONA-I-CARRASCO, J. y PUJADAS RÚBIES, I. Patrones espaciales de la segregación residencial en la Región Metropolitana de Barcelona: pautas de segregación de los grupos altos. Scripta Nova. Revista Electrónica de Geografía y Ciencias Sociales [En línea]. Barcelona: Universidad de Barcelona, 20 de diciembre de 2012, vol. 16, no 423. <http://www.ub.edu/geocrit/sn/sn-423.htm > [ 17 de Enero de 2018]. ISSN: 1138-9788.

RUBIALES, M. Territorio y crisis: impacto territorial de la crisis económica en las regiones metropolitanas de Madrid y Barcelona. Scripta Nova. Revista Electrónica de Geografía y Ciencias Sociales [En línea]. Barcelona: Universidad de Barcelona, 30 de Noviembre de 2016, vol. 20, num. 5-7. <http://www.ub.edu/geocrit/sn/sn-549-7. pdf.> [17 de Enero de 2018]. ISSN: 1138-9788.

SALOM, J., y FAJARDO, F. Cambios recientes en la estructura territorial sociodemográfica del área metropolitana de Valencia (2001-2011), Boletín de la Asociación de Geógrafos Españoles, 2017, nº 73, p. 123-147.

SASSEN, S. La ciudad global. Nueva York, Londres, Tokio. Eudeba, Buenos Aires, 1991

SASSEN, S. Ciudades en la economía global: enfoques teóricos y metodológicos. 
Revista EURE - Revista De Estudios Urbano Regionales, 1998, vol. 24, n71, p. 5-25.

SASSEN, S. Expulsiones. Brutalidad y complejidad en la economía global. Katz Ediciones. 2015

TEZANOS, J. F. La sociedad dividida: estructuras de clases y desigualdades en las sociedades tecnológicas. Madrid: Biblioteca Nueva, 2001.

VAN HAMME, G. "Social Cohesion in European Cities", en ESPON, Future Orientations for Cities. Final Scientific Repor, 2010.

VAN KEMPEN, R.; BOLT, G., Y VAN HAM, M. Neighborhood decline and the economic crisis: An introduction, Urban Geography, 2016, 37(5), p. 655-663.

VELTZ, P. Mundialización, ciudades y territorios. Barcelona, Ariel, 1999 


\section{Apéndice}

\section{Cuadro 1.A. Caracterización de los clústeres mediante variables}

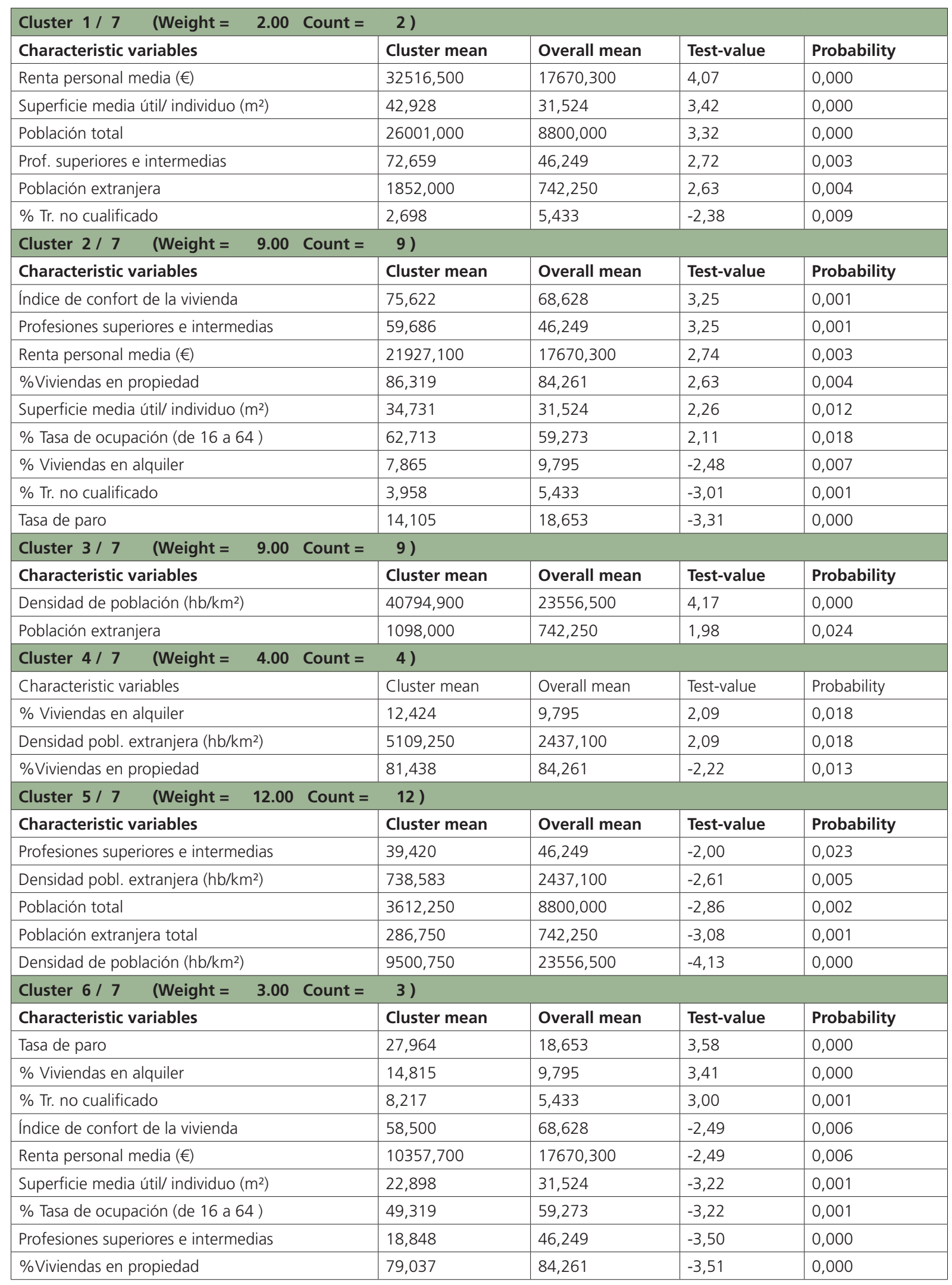




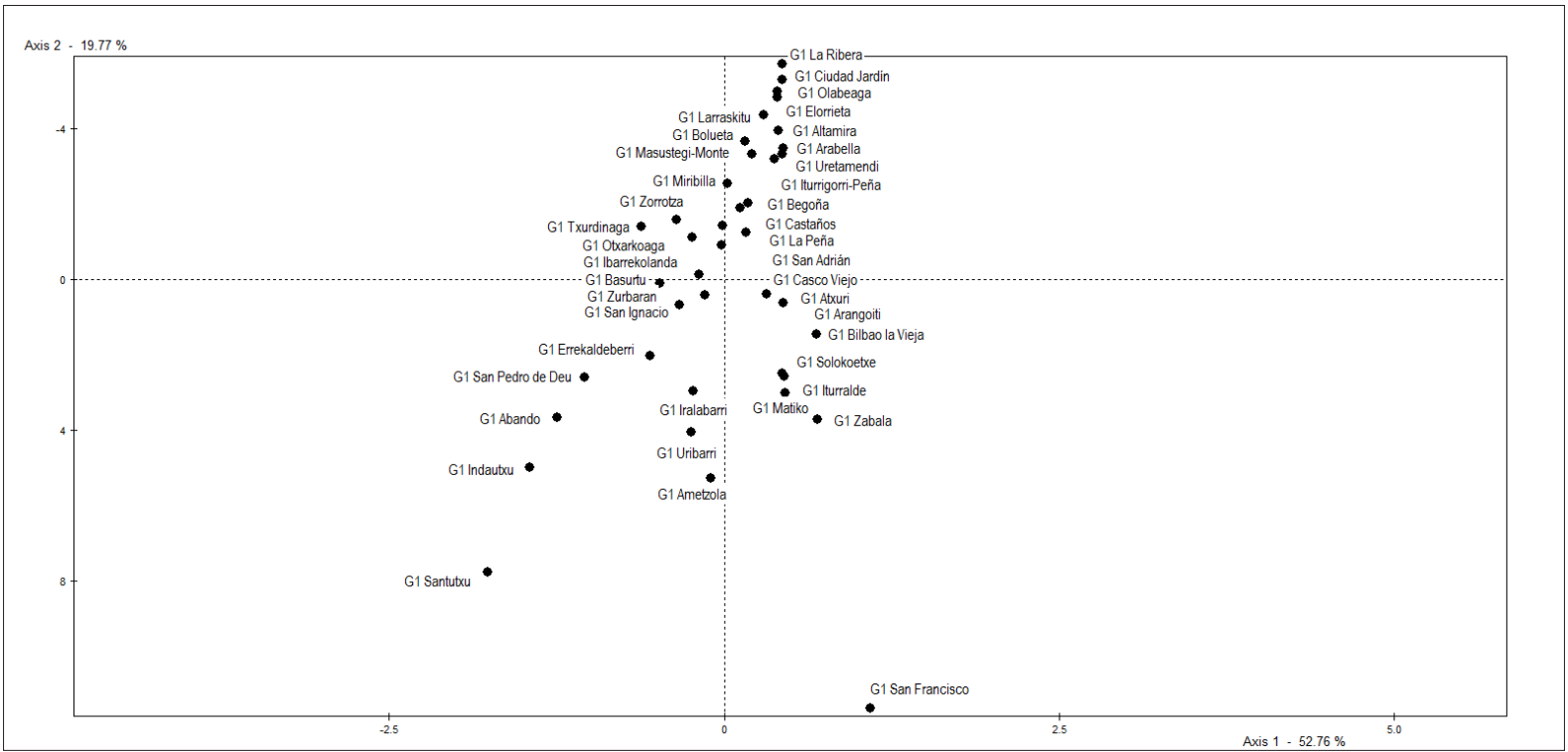

Figura 1A. Proyección de los individuos en el primer plano factorial (1-2). Dimensión demográfica ${ }^{48}$

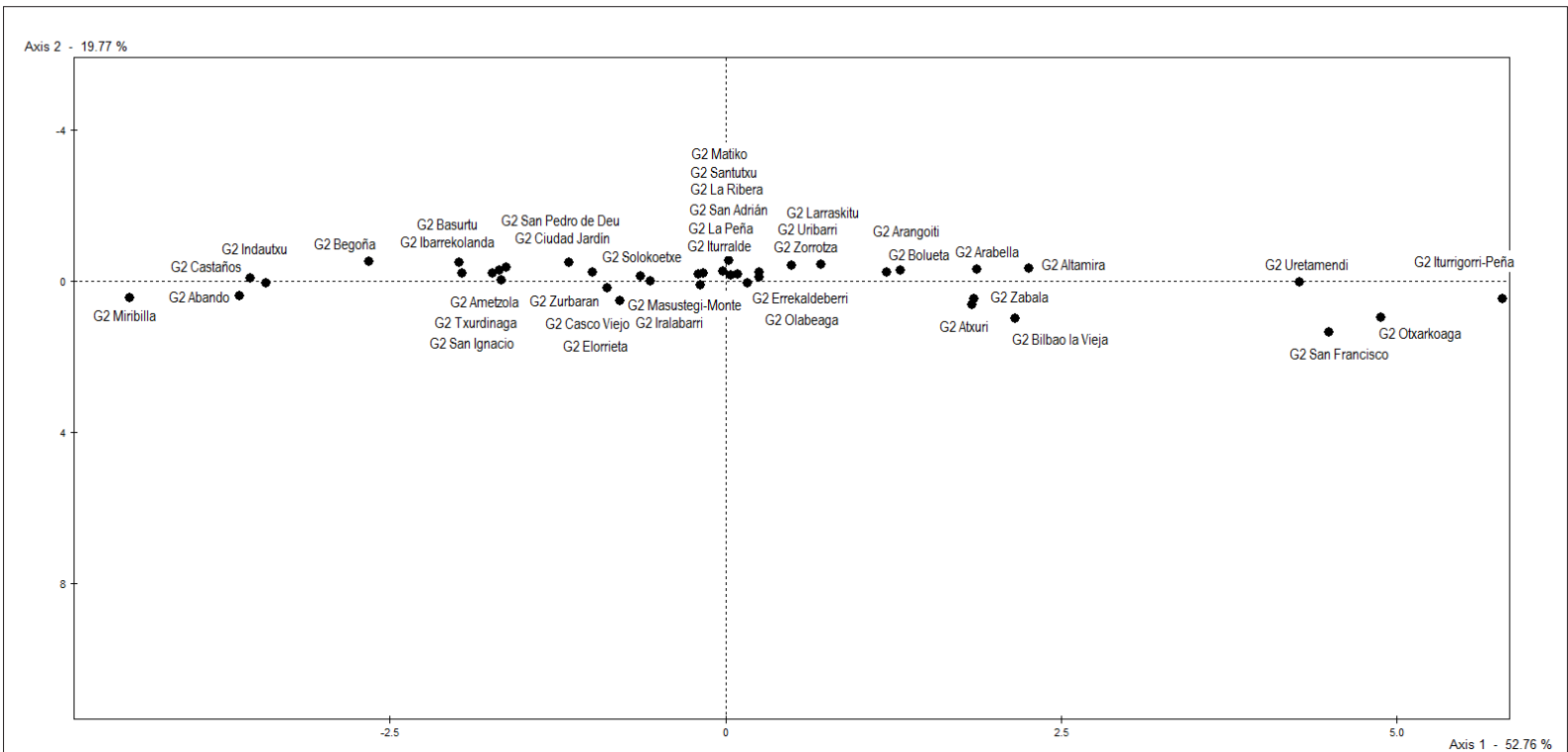

Figura 2A. Proyección de los individuos en el primer plano factorial (1-2). Condición socio-económica

48 En las Figuras 1A - 4A se proyectan (sobre el primer plano factorial del análisis global) los barrios desde la perspectiva de cada uno de los grupos de variables, respectivamente. Nótese que la escala de los ejes en estas Figuras es más amplia que en la Figura 3, en la que se proyectaban los individuos medios. 


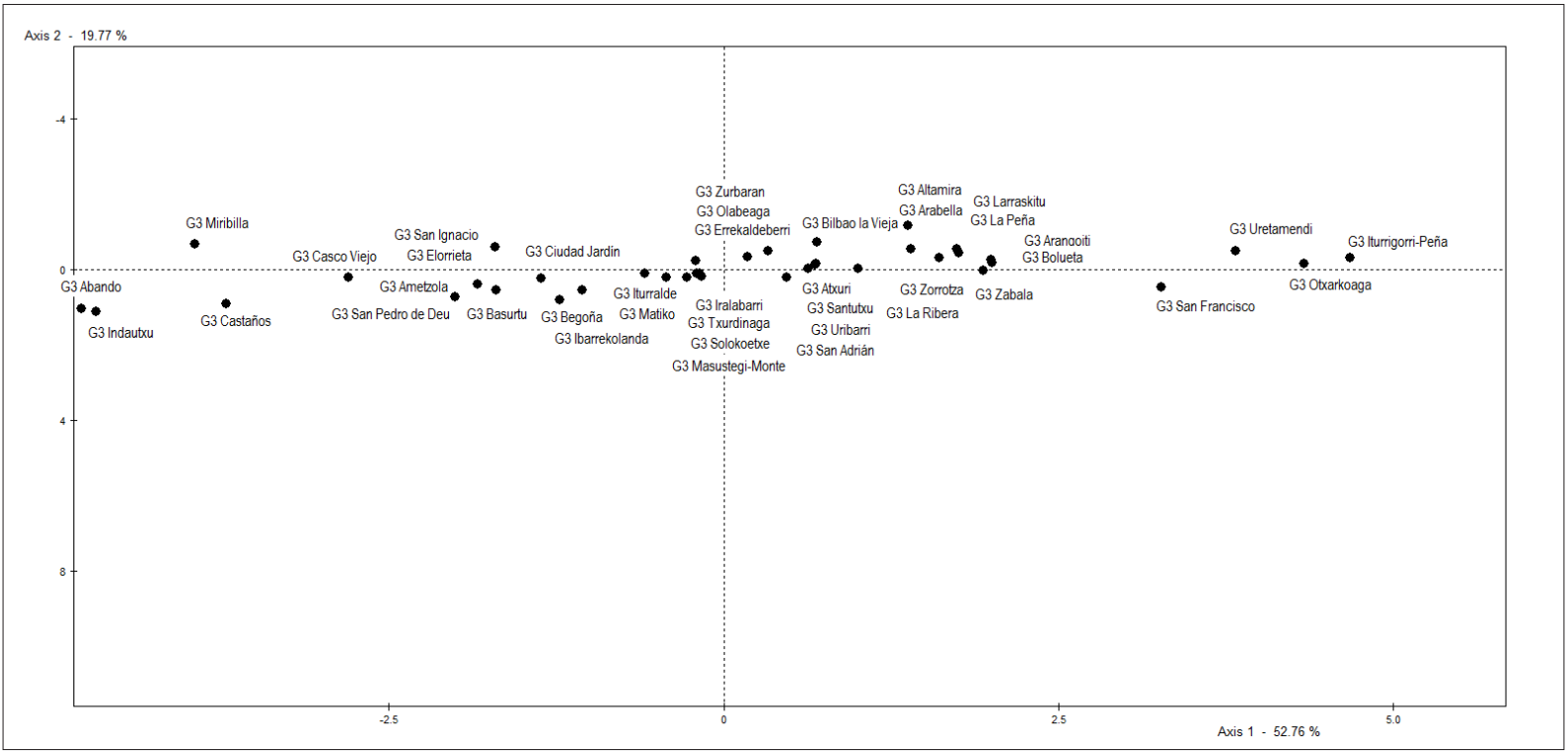

Figura 3A. Proyección de los individuos en el primer plano factorial (1-2). Características de la vivienda

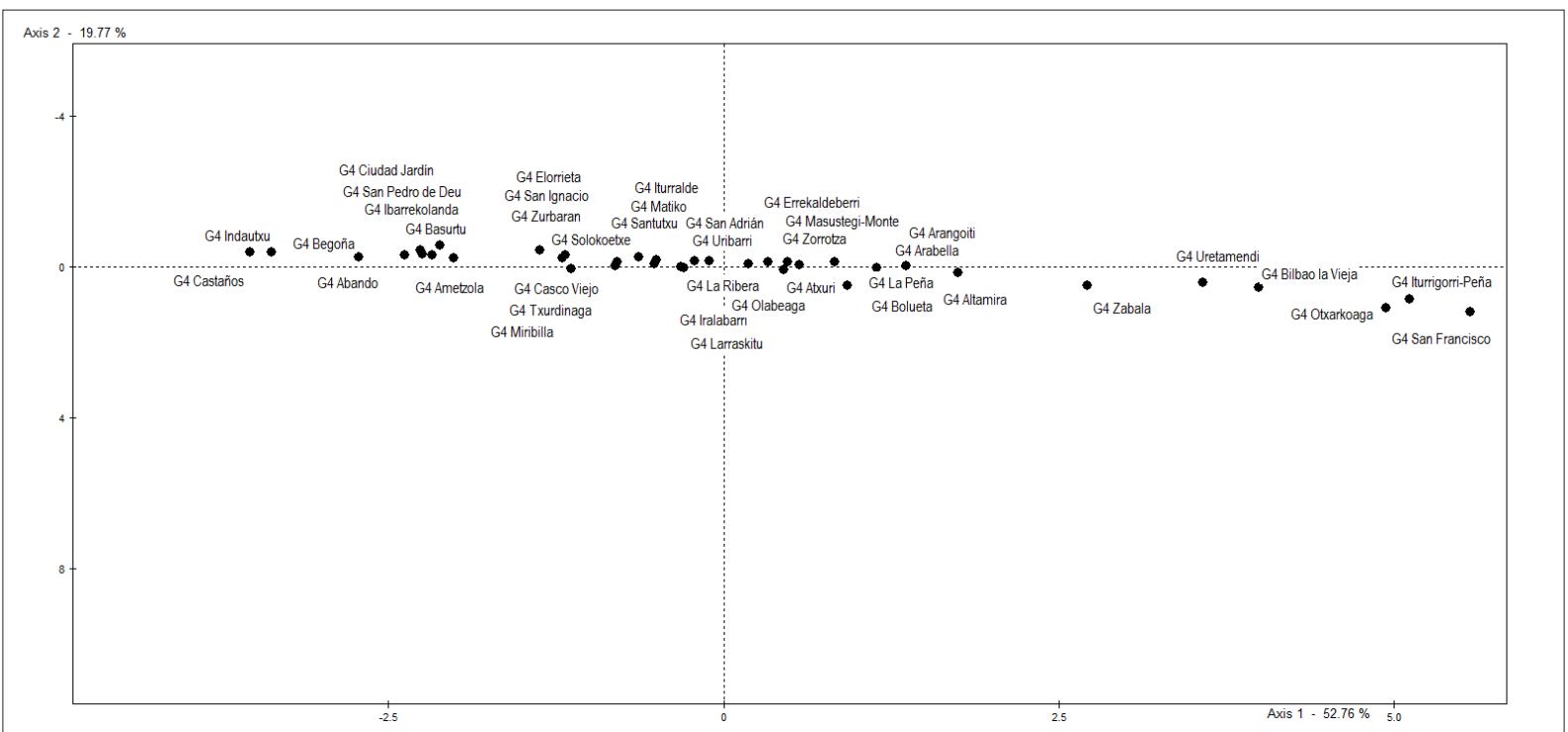

Figura 4A. Proyección de los individuos en el primer plano factorial (1-2). Cohesión social

(c) Copyright: Amaia Altuzarra Artola, Irantzu Álvarez González, Elena Martínez Tola, Arantxa Rodríguez Álvarez, 2018

(C) Copyright: Scripta Nova, 2018

Ficha bilbiográfica:

ALTUZARRA ARTOLA, Amaia; ÁLVAREZ GONZÁLEZ, Irantzu; MARTÍNEZ TOLA, Elena; RODRÍGUEZ ÁLVAREZ, Arantxa. Diferenciación socio-espacial urbana: mapa sintético de la estratificación de los barrios de Bilbao. Scripta Nova. Revista Electrónica de Geografía y Ciencias Sociales. Barcelona: Universidad de Barcelona, 1 de septiembre de 2018, vol. XXII, no 601[ISSN: 1138-9788] 
